\title{
The Strategic Use of Seller Information in Private-Value Auctions
}

\author{
Todd R. Kaplan \\ Department of Economics \\ Ben-Gurion University, \\ Center for Rationality \\ Hebrew University, Jerusalem
}

\author{
Shmuel Zamir* \\ Department of Statistics, \\ Center for Rationality \\ Hebrew University, Jerusalem, \\ CREST/LEI
}

July 2, 2000

\begin{abstract}
In the framework of a first-price private-value auction, we study the seller as a player in a game with the buyers in which he has private information about their realized valuations. We find that depending upon his information, set of signals, and commitment power he may strategically transmit messages to buyers in order to increase his revenue. In an environment where the seller knows the rankings and lacks any commitment power, we find that the seller is unable to exploit his information. However, in an environment where the seller knows the realized valuations and can credibly announce either the true rankings or the true values (or announce nothing at all) but cannot commit as to which of these truthful messages to announce, then it is indeed possible to increase his revenue. If the seller, in addition, can commit to the full signaling strategy, then his expected revenue will be even higher. We believe that this line of research is fruitful for both better understanding behavior in auctions and finding paths to higher seller revenue.
\end{abstract}

\section{Introduction}

The standard model of a private-value auction assumes that while there is common knowledge of the joint distribution of valuations, buyers have private infor-

${ }^{*}$ We gratefully acknowledge the support of CREST/LEI whose hospitality we enjoyed during the development of this paper. Partial support from EU-TMR ENDEAR project(FMRX-CT980238) is also acknowledged. We also wish to thank seminar participants at various universities especially Jacques Cremer, John Moore, Marco Ottaviani, Bradley Ruffle, Reinhard Selten, Jean Tirole, and Avi Weiss. 
mation about their own valuations. In this paper, we investigate the case where the seller is also informed about the buyers' private valuations. This can occur if either the seller possesses information about the buyers' types or information about the object's attributes.

A seller may have private information about the buyers' types if he simply possesses more information than one buyer about another buyer's type. ${ }^{1}$ For example, this may occur when the bidders' identities are known only to the seller or if previous interactions revealed information (such as losing bids) only to the seller. It may also occur if, from experience, the seller is better at judging types of buyers than the buyers are at judging each others' types.

Knowledge about particular attributes of the object for sale is a second source of seller information. For example, a database programmer (the seller) knows both Oracle and Sybase, while O.com (buyer 1) needs an Oracle programmer and S.com (buyer 2) needs a Sybase programmer. Each company is only able to judge the value of the programmer's skills that are relevant to its needs, whereas the programmer can evaluate his worth to each of the companies. Thus, the seller knows the object's worth to each buyer and each buyer only knows his own valuation for that object. Studying our scenario of seller information may have additional implications. If possessing private information proves valuable to the seller, he may either seek to obtain information that he lacks or attempt to prevent the discovery of his information by others. ${ }^{2}$

Once we endow the seller with private information, we must include him as a player in a game and consider the strategic consequences of possible actions. This can be examined in either the framework of mechanism design as we do in a subsequent paper (Kaplan and Zamir, 2000) or within a specific auction design as we do here. The latter would be more appropriate when either it is illegal to change the auction design or there is a high cost to do so. ${ }^{3}$ This may occur if the seller is uncertain about the type of his information at the time of auction design.

Possible specific auction designs to consider studying are those most com-

\footnotetext{
${ }^{1}$ This type need not be the precise valuation. For example, a buyer's type may be a preference for artwork by Picasso. This may indicate that for a particular work by Picasso he is more likely to have a higher valuation than a buyer without such preference.

${ }^{2}$ The Internet auction company Ubid hides not only losing bids but the sales price the instant an auction closes. Other sites such as Auctionwatchers try to obtain and compile such information.

${ }^{3}$ Changing the design may be illegal if, for instance, the government declares that a firstprice auction must be used or if the sale of the object is delegated to an auctioneer, the owner requests the use of a particular design from fear of collusion between the auctioneer and a buyer. It may also be impossible to change the design since such a design is a naturally occurring part of the environment, such as the database programmer eliciting job offers. It may be costly to change the design for both the buyers and the seller. For instance, an Internet auction company must implement the new design (rewrite software) and the buyers would need to learn the new rules.
} 
monly used: English, second-price sealed-bid, first-price sealed-bid, and Dutch. In an English, private-value auction, bidding up to one's value is a dominant strategy independent of what additional information one possesses. In a second-price sealed-bid auction, again a dominant strategy exists independent of additional information. This leaves us with a first-price sealed-bid (or the strategically equivalent Dutch auction), which is the simplest case of a commonly used mechanism where, as we shall see, the strategic use of seller information has non-trivial implications. This is the subject of the present paper.

Some evidence of these implications is provided by Landsberger, et al. [1998] (henceforth LRWZ), who consider a standard first-price auction with two buyers in which the rankings of the buyers' valuations are common knowledge. They find that when the buyers' valuations are drawn from a uniform distribution, both buyers bid more aggressively in equilibrium than they would have without this information, and consequently, the seller's revenue is higher. Although for this result the seller is not assumed to know the rankings, it raises the interesting question of whether a seller with such private information can increase revenue by transmitting some of his information to the buyers, inducing an equilibrium similar to LRWZ. In answering this question, we must consider several properties of the environment: the seller's information, available set of messages and commitment power.

Using LRWZ as a baseline, we first examine an environment in which the seller knows the rankings and can publicly announce a message as to which buyer has the highest value. Although a seller who knows the rankings will typically know more, we consider this example to gain insight into the role of commitment power in this context. In fact, we find that when the seller lacks commitment power (the ability to commit to sending messages based upon his information), he cannot profit from his information and his lack of credibility causes him to send a message with no meaning that is ignored. This holds for any number of public messages. In contrast, if the seller could fully commit to transmitting the true rankings, he can gain, as indicated by the results of LRWZ. Their results also imply that if the seller could commit to either transmit the true rankings or not (send a 'white' signal), he would also be able to increase his revenue (by transmitting the true rankings).

We thus develop the notion of 'partial truthful commitment' according to which the seller is constrained to send a 'truthful' message, but has several such truthful messages to choose from. For example, when the seller knows that buyer 1 has a valuation of .2 and buyer 2 has a valuation of .4, he can transmit (publicly) the message "buyer 2 has the highest valuation." He can also transmit the message "buyer 1 has a valuation of .2 and buyer 2 has a valuation of .4." In addition, he can refrain from sending any signal (equivalently send a "white signal'). However, he cannot commit to which of the three options he will choose. Although it may sound cryptic, this form of commitment seems reasonable. In general, if someone lies, it can be detected at some future date, perhaps an audit 
may reveal the truth. A lair would be subject to charges of fraud; however, not revealing all the information one possesses would not be punishable or at least much harder to prevent (although in a court of law, one swears to tell the whole truth and nothing but the truth). Such a form of commitment was first used in the Persuasion Game (Milgrom [1981]). In this game, a salesman reports the quality of a good (represented by a real number) to a buyer. His report (a subset of the real numbers) is restricted to contain the actual quality (known to the salesman).

To examine such a model, we consider the case where the seller knows the true valuations and can transmit the rankings (partial information), the valuations (the entire information), or a 'white' signal (no information). In this environment with such partial commitment, we show that the seller can still gain from his information. In particular, we find that the highest revenue generating equilibrium is that in which the seller sends the rankings when the low-value buyer has a value that is less than a fraction of the high-value buyer's value (half with a uniform distribution) and otherwise sends the values. Such an equilibrium is efficient.

Clearly any revenue achieved with partial truthful commitment can also be achieved with full commitment (i.e. by committing to use a certain signaling strategy). Thus, the next question is whether the seller can do strictly better if he has full commitment power. Previously, we saw that in the case where the seller knows only the rankings, both full and partial truthful commitment are equivalent. However, we show that this is not always the case by providing a strategy in the previous model (in which the seller knows the values) that by committing to use it, the seller induces a strictly higher expected revenue.

These results differ from two important results from earlier auction theory literature. First, the efficiency of the (highest revenue) partial commitment equilibrium violates the revenue equivalence of efficient auctions (Myerson [1981], Riley and Samuelson [1981]). Second, the strategy studied under full commitment is one of only partial revelation of seller information. This generates higher revenue than full revelation, in contrast to the Milgrom and Weber model [1982a, 1982b] in which full revelation of the seller's information provides the highest revenue.

Furthermore, this framework provides another insight into Strategic Information Transmission that is different from Crawford and Sobel [1982]. We note that in the Crawford-Sobel model information is partially disclosed due to the lack of commitment power on the part of the sender; under full commitment there is full revelation. In our model, partial disclosure of information takes place even when full commitment power is available, while nondisclosure occurs in the absence of commitment power.

The paper proceeds as follows. In Section 2, we introduce the levels of commitment and the subsequently used notation. In Section 3, we show that a seller without commitment power cannot benefit from his knowledge of the rankings. We then examine the environment with the seller possessing full knowledge of 
the values and present the results of partial commitment and full commitment in Sections 4 and 5 respectively. In Section 6, we compare the different cases of our model and discuss their relationship to the works of Milgrom and Weber [1982] and Crawford and Sobel [1982]. We conclude in Section 7.

\section{General Environment}

Our investigation of the seller's strategic use of private information is based upon the following three-player game (with the seller and the two buyers as players), which is a standard Harsanyi game of incomplete information. The data of the game consists of the sets of valuations $V_{1}$ and $V_{2}$, a cumulative probability distribution $F$ on $V=V_{1} \times V_{2}$, a partition $\pi$ of $V$, and an alphabet $A$ (a set of signals). The game is played as follows.

- Nature chooses the buyers' valuations (types) $v=\left(v_{1}, v_{2}\right) \in V$ according to the distribution $F$.

- Buyer 1 is informed of $v_{1}$, buyer 2 is informed of $v_{2}$, and the seller is informed of $\pi(v)$, the partition element of $\pi$ containing $v$.

- The seller announces publicly ${ }^{4}$ a message from his alphabet $A$.

- The buyers submit sealed bids $b_{1}$ and $b_{2}$.

- The buyer with the highest bid gets the object and pays his bid to the seller.

In this game, we represented the seller's information about the buyers' valuations as a partition $\pi$ of the buyers' type set $V$. The following are examples of such a partition.

1. $\pi=\{\{v\} \mid v \in V\}$ corresponds to a seller who knows the precise valuations (we refer to this $\pi$ as the maximal information).

2. $\pi=\{V\}$ corresponds to a seller with no private information about the valuations.

3. $\pi=\{L, H\}$, where $L=\left\{v=\left(v_{1}, v_{2}\right) \mid v_{1}<v_{2}\right\}$ and $H=\left\{v=\left(v_{1}, v_{2}\right) \mid v_{1} \geq\right.$ $\left.v_{2}\right\}$, corresponds to a seller who knows the rankings. ${ }^{5}$

\footnotetext{
${ }^{4}$ In further work, we also consider the possibility that the seller sends private messages $a_{1}$ and $a_{2}$ to buyers 1 and 2 , respectively.

${ }^{5}$ It is arbitrary, which element of the partition contains $v_{1}=v_{2}$ since in this paper, we consider only continuous distributions on $V$.
} 


\section{$2.1 \quad$ Levels of Commitment}

We continue to describe the environment by formally defining the levels of commitment that were mentioned in the introduction. Since some of the difference between the levels of commitment hinges on whether or not what the seller says is a 'true' statement. Before describing these levels of commitment we must define what we mean by 'truth' and 'truth-telling' strategies.

The seller's alphabet of signals is void of meaning without an interpretation function $I$, which assigns to any message a meaningful statement about the state of the world. Formally, let $\mathcal{P}(V)$ be the set of all subsets (power set) of $V$, then an interpretation function is: $I: A \rightarrow \mathcal{P}(V)$. This interpretation function is part of the environment and cannot be chosen or influenced by the seller. ${ }^{6}$

For example, if the information is maximal, $\pi=\{\{v\} \mid v \in V\}$, and the alphabet is $A=\{a, b\}$, then an interpretation function of $I(x)= \begin{cases}v_{1}<v_{2} & \text { if } x=a \\ v_{1} \geq v_{2} & \text { if } x=b\end{cases}$ implies that the statement of $a$ means that buyer 2 has the highest value and the statement of $b$ means that buyer 1 has the highest value.

The set of pure strategies (of the seller) is $S=\{s: \pi \rightarrow A\}$, or equivalently, $S=\{s: V \rightarrow A \mid s$ is $\pi$-measurable $\}$. The set of mixed strategies is $\Sigma_{s}=\triangle(S)$ (the set of probability distributions on $S$ ). The set of truth-telling pure strategies $S^{t}=\{s \in S \mid I(s(v)) \supseteq \pi(v), \forall v \in V\}$, while the set of truth-telling mixed strategies is $\Sigma_{s}^{t}=\triangle\left(S^{t}\right)$. (For instance, the only truth-telling strategy in the previous example would be to send $a$ if $v_{1}<v_{2}$ and $b$ if $v_{1} \geq v_{2}$.)

We now introduce four variants of games to capture the various levels of commitment power the seller may have. To avoid duplication we shall speak only of sets of (seller's) mixed strategies $\Sigma_{s}$ and $\Sigma_{s}^{t}$ and consider the pure strategy sets $S$ and $S^{t}$ as their subsets, respectively.

- A situation of no commitment corresponds to a game in which the seller's strategy set is $\Sigma_{s}$.

- A situation of partial truthful commitment is described by a game in which the seller's strategy set is $\Sigma_{s}^{t}$.

- A situation of full truthful commitment is described by a Stackelberg leadership game in which the seller can credibly commit to choosing from a nonempty, truthful strategy set $\Sigma_{s}^{c t} \subset \Sigma_{s}^{t}$, where this restriction is commonly known by the buyers.

\footnotetext{
${ }^{6}$ If the seller instead could choose the interpretation function, the difference between the levels of commitment (other than no commitment) would be greatly diminished as the propositions in this section show. Also, future research may have the interpretation function emerging endogenously as part of the equilibrium of an evolutionary model.
} 
- A situation of full commitment is described by a Stackelberg leadership game, as before, where the seller can credibly restrict himself to a nonempty strategy set $\Sigma_{s}^{c} \subset \Sigma_{s}$ commonly known by the buyers.

Loosely speaking, the above classification captures the four possibilities of available commitment power: whether or not the seller must use truthful messages, and whether or not he can credibly restrict his signaling strategy set. Formally, the first two games differ, as do the last two games, by the seller's strategy set, while the first two differ from the last two by their game structure.

The following example should provide insight into the definitions. The seller has information partition $\pi=\{w, x, y, z\}$ (which values are in which partition element is not germane to the example). The alphabet is $A=\{a, b, c, d\}$. The interpretation of this alphabet, $I$, is $I(a)=\{w\}, I(b)=\{x\}, I(c)=\{w, x\}$ and $I(d)=\{y, z\}$. Under no commitment, there would be no restrictions on the seller's strategy. Under partial truthful commitment, the seller would be restricted to sending $a$ or $c$ when informed of $w$, sending $b$ or $c$ when informed of $x$, and sending $d$ when informed of $y$ or $z$. Under full truthful commitment, the seller has the ability to credibly commit to choosing from a restricted strategy set of truthful strategies. For example, he could restrict himself to sending $a$ when $w, c$ when $x$, and $d$ when $y$ or $z$. Under full commitment, the restricted set may include non-truthful strategies. He can, for example, restrict himself to sending signal $a$ when $w$, signal $b$ when $x$, signal $c$ when $y$ (which is not truthful), and signal $d$ when $z$.

As mentioned in the introduction, the idea of 'partial truthful commitment' is employed in the Persuasion game (Milgrom [1981] and Milgrom \& Roberts [1986]) where "truth" is captured in the form of allowing the seller of the good to send a message in the form of a set (in the quality space $[0,1]$ ) containing the true quality. A similar notion appears later in Seidmann and Winter [1997] where the "truth" telling is captured by making the available set of signals state dependent (so that lying, which does not exist explicitly in their model, would correspond to a message which is not available in that state of information). In our model, the notion of "truth" is captured by the interpretation function.

\subsection{Equilibrium}

We denote the seller's strategy set as before and we let $\Sigma_{i}: V_{i} \times A \longrightarrow \triangle\left[R^{+}\right]$be buyer $i$ 's strategy set, where $i$ is in the set of buyers $B$. The various games have strategy sets defined as follows:

- The no commitment game $\Gamma_{n}$ has strategy sets $\left(\Sigma_{s},\left(\Sigma_{i}\right)_{i \in B}\right)$.

- The partial truthful commitment game $\Gamma_{p t}$ has strategy sets $\left(\Sigma_{s}^{t},\left(\Sigma_{i}\right)_{i \in B}\right)$.

- The full commitment game $\Gamma_{f}$ has strategy sets $\left(\Sigma_{s},\left(\Sigma_{i}^{*}\right)_{i \in B}\right)$, where $\Sigma_{i}^{*}=$ $\left\{\sigma_{i}^{*}: \Sigma_{s} \rightarrow \Sigma_{i}\right\}$. 
- The full truthful commitment game $\Gamma_{f t}$ has strategy sets $\left(\Sigma_{s}^{t},\left(\Sigma_{i}^{t *}\right)_{i \in B}\right)$, where $\Sigma_{i}^{t *}=\left\{\sigma_{i}^{t *}: \Sigma_{s}^{t} \rightarrow \Sigma_{i}\right\}$.

Denote $\Sigma_{B}=\times_{i \in B} \Sigma_{i}, \Sigma_{B}^{*}=\times_{i \in B} \Sigma_{i}^{*}$, and the payoff of player $i$ (in $B \cup\{s\}$ ) as $H_{i}: \Sigma_{s} \times \Sigma_{B} \rightarrow R$. Also, by the slight abuse of notation, we write $H_{i}\left(\sigma_{s}, \sigma_{B}^{*}\right)$ for $\sigma_{B}^{*} \in \Sigma_{B}^{*}$ to mean $H_{i}\left(\sigma_{s}, \sigma_{B}^{*}\left(\sigma_{s}\right)\right)$.

Accordingly (denoting, as usual $\sigma_{-i}$ and $\sigma_{-i}^{*}$ elements of $\Sigma_{-i}=\times_{j \in B \backslash\{i\}} \Sigma_{j}$ and $\Sigma_{-i}^{*}=\times_{j \in B \backslash\{i\}} \Sigma_{j}^{*}$ respectively), a Nash equilibrium in $\Gamma_{n}$ is a $\sigma=\left(\sigma_{s}, \sigma_{B}\right)$ such that $H_{i}(\sigma) \geq H_{i}\left(\tilde{\sigma}_{i}, \sigma_{-i}\right)$ for all $\tilde{\sigma}_{i} \in \Sigma_{i}$ and $i \in B \cup\{s\}$. A sequential equilibrium in $\Gamma_{n}$ is a Nash equilibrium $\sigma=\left(\sigma_{s}, \sigma_{B}\right)$ such that for all $i \in B, a$ in $A$ and $v_{i}$ in $V_{i}$, there exists a $\tilde{\sigma}_{s}$ in $\Sigma_{s}$ such that $H_{i}\left(\tilde{\sigma}_{s}, \sigma_{B} \mid a, v_{i}\right) \geq$ $H_{i}\left(\tilde{\sigma}_{s}, \tilde{\sigma}_{i}, \sigma_{-i} \mid a, v_{i}\right)$ for all $\tilde{\sigma}_{i} \in \Sigma_{i}$ and $i \in B$. Also, a subgame-perfect equilibrium in $\Gamma_{f}$ is a $\sigma=\left(\sigma_{s}, \sigma_{B}^{*}\right)$ such that $H_{i}\left(\tilde{\sigma}_{s}, \sigma_{B}^{*}\right) \geq H_{i}\left(\tilde{\sigma}_{s}, \tilde{\sigma}_{i}^{*}, \sigma_{-i}^{*}\right)$ for all $i \in$ $B, \tilde{\sigma}_{i}^{*} \in \Sigma_{i}^{*}$, and $\tilde{\sigma}_{s} \in \Sigma_{s}$ and $H_{s}(\sigma) \geq H_{s}\left(\tilde{\sigma}_{s}, \sigma_{B}^{*}\left(\tilde{\sigma}_{s}\right)\right)$ for all $\tilde{\sigma}_{s} \in \Sigma_{s}$. A sequential equilibrium for $\Gamma_{p t}$ and a subgame-perfect equilibrium for $\Gamma_{f t}$ are defined in a similar way.

As can be seen from these definitions, the (only) subgames considered in $\Gamma_{f}$ and $\Gamma_{f t}$ are those resulting from fixing the strategy of the seller to $\sigma_{s} \in \Sigma_{s}$. In $\Gamma_{n}$ and $\Gamma_{p t}$, there are no such subgames and we resort to a notion of sequential equilibria.

Throughout this paper, an equilibrium in $\Gamma_{n}$ (or in $\Gamma_{p t}$ ) stands for a sequential equilibrium and an equilibrium in $\Gamma_{f}\left(\right.$ or $\left.\Gamma_{f t}\right)$ stands for subgame-perfect equilibrium.

\subsection{General Implications}

Before analyzing specific environments, it is worthwhile to see what general results can already be derived from the above definitions. In what follows, we provide some weak inequalities among the different levels of commitment. In subsequent sections, we give specific examples showing that these inequalities can be strict.

We use the following definition to compare games, using the appropriate equilibrium concept for each.

Definition: Given two games $\Gamma$ and $\tilde{\Gamma}$ and a player $i$ in both of them, let $H_{i}$ and $\tilde{H}_{i}$ be the payoff functions of player $i$ in $\Gamma$ and $\tilde{\Gamma}$ respectively. We say that $i$ does better in $\Gamma$ than in $\tilde{\Gamma}$ (and write $\Gamma \succ_{i} \tilde{\Gamma}$ ) if:

1. For any equilibrium $\sigma$ of $\Gamma$, there is an equilibrium $\tilde{\sigma}$ of $\tilde{\Gamma}$ such that $H_{i}(\sigma) \geq \tilde{H}_{i}(\tilde{\sigma})$.

2. For any equilibrium $\tilde{\sigma}$ of $\tilde{\Gamma}$, there is an equilibrium $\sigma$ of $\Gamma$ such that $H_{i}(\sigma) \geq \tilde{H}_{i}(\tilde{\sigma})$.

Proposition 2.1 The seller's preference on the various games satisfy the following relationships:

$$
\Gamma_{f} \succ_{s} \Gamma_{f t} \succ_{s} \Gamma_{p t} \text { and } \Gamma_{f} \succ_{s} \Gamma_{n}
$$


Proof: Let $\sigma=\left(\sigma_{s}, \sigma_{B}\right)$ be a sequential equilibrium of $\Gamma_{n}$. For $i \in B$, consider the set $\Sigma_{i}^{*}(\sigma)$ of buyer strategies in $\Gamma_{f}$ that have the same subgame behavior in subgame $\sigma_{s}$, that is,

$$
\Sigma_{i}^{*}(\sigma)=\left\{\tilde{\sigma}_{i}^{*} \in \Sigma_{i}^{*} \mid \tilde{\sigma}_{i}^{*}\left(\sigma_{s}\right)=\sigma_{i}\right\}
$$

There exists a subgame-perfect equilibrium $\tilde{\sigma}$ in $\Gamma_{f}$ such that $\tilde{\sigma}_{B} \in \Sigma_{B}^{*}(\sigma)$. (Substitute the payoffs of the $\sigma_{s}$ branch into the tree. There is a subgame-perfect equilibrium of this new tree; thus, there is one in the old tree as well.) Notice that this implies that $H_{s}^{f}(\tilde{\sigma}) \geq H_{s}(\sigma)$ (to avoid confusion, we write $H_{s}^{f}$ as the payoff in $\left.\Gamma_{f}\right)$. Thus, for any equilibrium $\sigma$ in $\Gamma_{n}$, there is an equilibrium $\tilde{\sigma}$ in $\Gamma_{f}$ with weakly higher payoff to the seller (establishing condition 2 for $\Gamma_{f} \succ_{s} \Gamma_{n}$ ).

Let $E_{n}$ be the set of sequential equilibria of $\Gamma_{n}$, and let

$$
\sigma=\arg \min _{\tilde{\sigma} \in E_{n}} H_{s}(\tilde{\sigma})
$$

For any subgame-perfect equilibrium $\sigma^{*}$ of $\Gamma_{f}, H_{s}^{f}\left(\sigma^{*}\right) \geq H_{s}(\sigma)$. Thus, for any subgame-perfect equilibrium in $\Gamma_{f}$, there is an equilibrium in $\Gamma_{n}$, namely $\sigma$, with weakly lower payoff for the seller. This concludes the proof of $\Gamma_{f} \succ_{s} \Gamma_{n}$. In a similar manner, we can prove that $\Gamma_{f} \succ_{s} \Gamma_{p t}$ and $\Gamma_{f t} \succ_{s} \Gamma_{p t}$.

It is left to prove that $\Gamma_{f} \succ_{s} \Gamma_{f t}$. This follows from the observation that the seller's strategy set is larger in $\Gamma_{f}$ than in $\Gamma_{f t}$ : if there is an equilibrium $\left(\sigma_{s}, \sigma_{B}^{t *}\right)$ in $\Gamma_{f t}$, there must be an equilibrium $\left(\sigma_{s}^{\prime}, \sigma_{B}^{\prime *}\right)$ in $\Gamma_{f}$ such that $\sigma_{B}^{\prime *}\left(\sigma_{s}\right)=\sigma_{B}^{t *}\left(\sigma_{s}\right)$. For this equilibrium, either $\sigma_{s}=\sigma_{s}^{\prime}$ (and the seller receives the same payoff in both equilibria) or the seller receives (weakly) more in $\left(\sigma_{s}^{\prime},{\sigma_{B}^{\prime *}}^{*}\right)$ than in $\left(\sigma_{s}, \sigma_{B}^{t *}\right)$, establishing condition 2 for $\Gamma_{f} \succ_{s} \Gamma_{f t}$. In the other direction, the equilibrium $\left(\sigma_{s}, \sigma_{B}^{t *}\right)$ in $\Gamma_{f t}$ with the lowest seller payoff will pay the seller weakly less than any equilibrium $\left(\sigma_{s}^{\prime}, \sigma_{B}^{*}\right)$ in $\Gamma_{f}$, since the seller could choose $\sigma_{s}$ in $\Gamma_{f}$ and induce the path $\sigma_{B}^{*}\left(\sigma_{s}\right)$.

\section{$2.4 \quad$ Verifiability}

What may allow some form of commitment to a specific signaling strategy? A possible answer is the availability of some type of verification at a future point in time. That is, a seller can commit to a strategy set if eventually one can discover the seller's information and verify if the signal sent is consistent with that set of signaling strategies. In the following, we will formalize the definition of a verifiable strategy set and investigate the implications for the levels of commitment that we examine. In particular, we will show that our notion of partial-truthful commitment is reasonable since the truthful-strategy set is verifiable.

For $S^{c} \subset S$ and $v \in V$, define $S^{c}(v) \subset A$ by $S^{c}(v)=\left\{s(v) \mid s \in S^{c}\right\}$.

Definition: A set of (seller's) pure strategies $S^{c} \subset S$ is verifiable if for all $\tilde{s} \in S$, $\left\{\tilde{s}(v) \in S^{c}(v), \forall v \in V\right\} \Longrightarrow \tilde{s} \in S^{c}$. 
An example of a set of pure strategies which is not verifiable is the following. The seller has an information partition of two elements, $\pi_{1}$ and $\pi_{2}$, and has two signals, $a$ and $b$, at his disposal. Consider the following strategy set $S^{c}=\left\{s_{1}, s_{2}\right\}$, where $s_{1}\left(\pi_{1}\right)=a, s_{1}\left(\pi_{2}\right)=b, s_{2}\left(\pi_{1}\right)=b$ and $s_{2}\left(\pi_{2}\right)=a$. This set is not verifiable, since, for example, $s_{3}$ defined by $s_{3}\left(\pi_{1}\right)=s_{3}\left(\pi_{2}\right)=a$ satisfies $s_{3}(v) \in S^{c}(v)$ for all $v \in V$, yet $s_{3}$ is not in $S^{c}$.

Proposition 2.2 The set of pure truthful strategies, $S^{t}$, is verifiable.

Proof: For all $v \in V$,

$$
\begin{gathered}
S^{t}(v)=\left\{s(v) \mid s \in S^{t}\right\}=\left\{s(v) \mid s \in S, I\left(s\left(v^{\prime}\right)\right) \supset s\left(v^{\prime}\right), \forall v^{\prime} \in V\right\} \\
\subset\{s(v) \mid s \in S, I(s(v)) \supset s(v)\}
\end{gathered}
$$

Hence, $\left\{s(v) \in S^{t}(v), \forall v \in V\right\} \Rightarrow\{I(s(v)) \supset \pi(v), \forall v \in V\} \Rightarrow s \in S^{t}$.

We now make a comparable definition of verifiability for mixed strategies. Denoting by $\operatorname{Supp}(\sigma)$ the support of the (seller's) mixed-strategy $\sigma$, for $\sigma \in \Sigma$ and $v \in V$, define $\sigma(v)=\{s(v) \mid s \in \operatorname{Supp}(\sigma)\}$. For $\Sigma^{c} \subset \Sigma$ let $\Sigma^{c}(v)=\cup_{\sigma \in \Sigma^{c}} \sigma(v)$ and $\operatorname{Supp}\left(\Sigma^{c}\right)=\cup_{\sigma \in \Sigma^{c}} \operatorname{Supp}(\sigma)$.

Definition: A set of mixed strategies $\Sigma^{c} \subset \Sigma$ is $\Sigma$-verifiable if for all $\tilde{\sigma} \in \Sigma$, $\left\{\tilde{\sigma}(v) \subset \Sigma^{c}(v), \forall v \in V\right\} \Longrightarrow \tilde{\sigma} \subset \Sigma^{c}$.

Denoting by $C o n(D)$ the convex-hull of the set $D$, we have:

Proposition 2.3 A set $\Sigma^{c}$ of seller's mixed strategies is $\Sigma$-verifiable if and only if $\Sigma^{c}=\operatorname{Con}\left(S^{c}\right)$ where $S^{c}$ is verifiable.

Proof: Let $\Sigma^{c}$ be a $\Sigma$-verifiable set of mixed strategies. We show that $\Sigma^{c}=$ $\operatorname{Con}\left(\operatorname{Supp}\left(\Sigma^{c}\right)\right)$ and that $\operatorname{Supp}\left(\Sigma^{c}\right)$ is verifiable.

First, notice that $\Sigma^{c}$ is convex. This follows from the fact that if $\tilde{\sigma}$ is a (strict) convex combination of $\sigma_{1}$ and $\sigma_{2}$ then $\tilde{\sigma}(v)=\sigma_{1}(v) \cup \sigma_{2}(v)$, for all $v \in V$.

Second, the extreme points of $\Sigma^{c}$ are pure strategies. In fact, if $\Sigma^{c}$ is $\Sigma$ verifiable and $\sigma \in \Sigma^{c}$ then $s \in \Sigma^{c}$, for all $s \in S u p p(\sigma)$ (since $s(v) \in \sigma(v)$, for all $v \in V)$. Since clearly any $s \in \operatorname{Supp}\left(\Sigma^{c}\right)$ is an extreme point of $\Sigma^{c}$, it follows that $\Sigma^{c}=\operatorname{Con}\left(S^{c}\right)$, where $S^{c}=\operatorname{Supp}\left(\Sigma^{c}\right)$. It remains to prove that this $S^{c}$ is a verifiable set of pure strategies. This follows from noticing that $\Sigma^{c}(v)=S^{c}(v)$ for all $v \in V$ and hence: for all $\tilde{s} \in S$,

$\left\{\tilde{s}(v) \in S^{c}(v), \forall v \in V\right\} \Rightarrow\left\{\tilde{s}(v) \in \Sigma^{c}(v), \forall v \in V\right\} \Rightarrow \tilde{s} \in \Sigma^{c} \Rightarrow \tilde{s} \in \operatorname{Supp}\left(\Sigma^{c}\right)=S^{c}$.

To prove the 'if' part of the statement, let $S^{c}$ be a verifiable set of pure strategies. We want to show that $\operatorname{Con}\left(S^{c}\right)$ is $\Sigma$-verifiable, i.e. we have to show that for all $\tilde{\sigma} \in \Sigma,\left\{\tilde{\sigma}(v) \subset \operatorname{Con}\left(S^{c}\right)(v), \forall v \in V\right\}$ implies $\tilde{\sigma} \in \operatorname{Con}\left(S^{c}\right)$.

As noticed before: $\operatorname{Con}\left(S^{c}\right)(v)=S^{c}(v)$ and $\tilde{\sigma}(v)=\{\tilde{s}(v) \mid \tilde{s} \in \operatorname{Supp}(\tilde{\sigma})\}$. Therefore, we have $\left\{\tilde{\sigma}(v) \subset \operatorname{Con}\left(S^{c}\right)(v), \forall v \in V\right\}$ implies that for all $\tilde{s} \in \operatorname{Supp}(\tilde{\sigma})$, $\left\{\tilde{s}(v) \in S^{c}(v), \forall v \in V\right\}$. Since $S^{c}$ is verifiable, this implies $\tilde{s} \in S^{c}$ and hence $\tilde{\sigma} \in \operatorname{Con}\left(S^{c}\right)$. 
Corollary 2.1 The set of mixed truthful strategies $\Sigma^{t}$ is $\Sigma$-verifiable.

Proof: This follows from Proposition 2.3 since $\Sigma^{t}=\triangle\left(S^{t}\right)=\operatorname{Con}\left(S^{t}\right)$ and $S^{t}$ is verifiable by Proposition 2.2.

Corollary 2.2 No single strategy is $\Sigma$-verifiable unless it is a pure strategy.

In the general game-theoretical framework, one may ask whether a sender of a signal in a game would ever want to commit to a non-verifiable strategy. We can provide the answer with an example of such a case.

Consider a game with two receivers playing the following game:

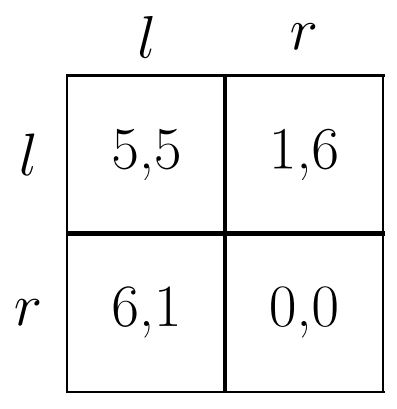

This game has two pure equilibria, $(l, r)$ and $(r, l)$ with payoffs $(1,6)$ and $(6,1)$, respectively, a mixed-strategy equilibrium with payoff $(3,3)$ (both play $(1 / 2,1 / 2))$ and a large set of correlated equilibria including one with payoff $(4,4)$ (probability $1 / 3$ for each of $(l, l),(l, r)$, and $(r, l))$. If the sender's payoff is the sum of both receiver's payoffs, he would have incentive to help them obtain a correlated equilibria. He cannot credibly use this correlated mixture of $(1 / 3(l, l)+$ $1 / 3(l, r)+1 / 3(r, l))$, since he prefers the choice of $(l, l)$ which gives him a payoff of 10 (as opposed to a payoff of 8). There is no method to verify that he was not mixing according to the correlated equilibrium even if most of the time he is sending the corresponding signals to obtain $(l, l)$ (unless of course in an infinitely repeated play setup where the mixed strategy can be statistically tested with arbitrarily large precision). We conclude that the sender would like to commit to the mixed non-verifiable signaling strategy leading to the correlated equilibrium.

In the classic way of treating correlated equilibria, the previous example would entail private signals. We now show that such correlated equilibria also exist when the sender is limited to a public message. Thus, a desire for commitment to non-verifiable exists for public signals as well. Take the previous game with the following modification. For each receiver, there is a coin flip seen only by him and the sender. The sender publicly mentions which action to take; however, a receiver does the action mentioned if his coin is heads and the other action if his 
coin is tails. The correlated equilibrium are analogous to those in the previous game, since, as before, each receiver believes the other receiver has a equal chance of choosing each action. ${ }^{7}$

In the following, we can formally see the above results. Each coin can have two possible outcomes: heads or tails. We define the set of outcomes as $C=\{h, t\}$. Each player has two possible moves: $l$ or $r$. His set of moves is $M=\{l, r\}$. The sender has an alphabet of private signals (recommendations) to send to the players $A=\{L, R\}$. The sender's pure strategy set is $S^{s}: C^{2} \rightarrow A^{2}$, while each player has the strategy set of $S^{p}: C \times A \rightarrow M$. Mixed strategies are defined as $\Sigma^{s}=\triangle\left(S^{s}\right)$ and $\Sigma^{p}=\triangle\left(S^{p}\right)$. Payoffs are defined by $h^{i}\left(\sigma^{s}, \sigma^{p 1}, \sigma^{p 2}\right)$

Let us define three sender pure strategies: $a, b$, and $c$. For strategy $a, a(h h)=$ $L L, a(t t)=R R, a(t h)=R L$ and $a(h t)=L R$. For strategy $b, b(h h)=L R$, $b(t t)=R L, b(h t)=L L$, and $b(t h)=R L$. For strategy $c, c(h h)=R L, c(t t)=$ $L R, c(t h)=L L$, and $c(h t)=R L$.

Let us define a player's strategy $e$ as follows: $e(h, L)=l, e(h, R)=r, e(t, L)=$ $r$, and $e(t, R)=l$.

Notice that if the sender commits to using strategy $1 / 3 a+1 / 3 b+1 / 3 c$, the players have an equilibrium using strategy $e$. Also note that without a commitment, the sender would have incentive to deviate to always sending $a$, which would result in the players playing one of the Nash equilibrium mentioned above.

\subsection{Connections among different levels of commitment power}

As it is intuitively clear, the interpretation function is crucial in determining the outcome of the game. The next two propositions show that a limited interpretation function can serve as a substitute of a commitment device in the partial and full truthful commitment cases.

Proposition 2.4 For each equilibrium with full commitment:

(i) There exists an interpretation function for which an outcome equivalent equilibrium exists with full truthful commitment.

(ii) When the equilibrium strategy set chosen in the first stage of full commitment is verifiable, there exists an interpretation function for which an outcome equivalent equilibrium exists with partial truthful commitment as well.

For each equilibrium with full truthful commitment:

(iii) When the equilibrium strategy set chosen in the first stage of full truthful commitment is verifiable, there exists an interpretation function for which an outcome equivalent equilibrium exists with partial truthful commitment.

\footnotetext{
${ }^{7}$ Future work to find an example where a player would want to commit to a nonsingleton set of non-verifiable strategies would be useful.
} 
Proof: Part (i) follows trivially by noting that any given full commitment equilibrium becomes a full truthful equilibrium if we change the interpretation function to $\tilde{I}(a)=V$, for all $a \in A$ (i.e. all messages are truthful).

To prove (ii), consider a full commitment equilibrium in which the seller restricts his strategy set to $S^{c}$ (a subset of pure strategies). Define an interpretation function $I$ as follows: for $a \in A$, let

$$
I(a)=\left\{\pi(v) \mid v \in V \text { and } a \in S^{c}(v)\right\}
$$

Then, by definition of $I, S^{c} \subset S^{t}$. We claim that if $S^{c}$ is verifiable, then $S^{c}=S^{t}$. In fact, let $\tilde{s} \in S^{t}$. This means that if $\{v \in V$ and $\tilde{s}(v)=a\}$, then $I(a) \supset \pi(v)$. This implies (by definition of $I$ ) that there exists an $s \in S^{c}$ with $s(v)=a$ and hence $\left\{\tilde{s}(v) \in S^{c}(v), \forall v \in V\right\}$. This then implies $\tilde{s} \in S^{c}$ since $S^{c}$ is verifiable. We conclude that $S^{t} \subset S^{c}$ and hence $S^{t}=S^{c}$.

Finally, part (iii) is shown in a similar manner as part (ii).

Corollary 2.3 For any verifiable set $S^{c}$ there exists an interpretation function I such that $S^{t}$ for that $I$ is equal to $S^{c}$.

The content of the last proposition is that the interpretation function can act as a form of substitute for commitment power. Consequently, within a specific framework a seller can benefit from having one interpretation function over another. For example, a seller may benefit from having an interpretation function become finer or coarser. We can see this when the seller has full information with full truthful commitment. Consider the case of two buyers with i.i.d. valuations uniformly distributed on $[0,1]$. When the only truthful statement is to state the values, the seller's revenue is $1 / 3$ as in the English auction. When there is only one truthful message that conveys no value, the seller's revenue is also $1 / 3$ as in a first-price auction. When there is a truthful statement that states the rankings, the seller's revenue is at least .3696 as in LRWZ.

This shows room for a strategic influence on the interpretation function. In a dynamic model, players (e.g. lawyers) may try to make the interpretation function more specific or less specific. In an evolutionary model, a language (represented by an interpretation function) may develop based on needs of players.

\section{When the Seller knows the Rankings and has no Commitment Power.}

We begin our analysis of auctions by examining the case when the seller has no commitment power, but has information as to which buyer has the highest value (the rankings). Here the seller has the same information that the buyers possess in LRWZ. Thus, we are able to ask whether or not a seller without commitment power can gain from having such information by communicating with the buyers. 
First, we assume that the seller has to publicly report the rankings. He thus has two possible actions: to lie or tell the truth. We show that in this situation the seller cannot gain from his information. Second, we show that if the seller can send any public message based upon his information, he can still not gain.

Consider a standard first-price sealed-bid private-value auction with valuations drawn independently from a continuous differentiable distribution on $[0,1]$. In the Harsanyi framework of games with incomplete information, this is a game with state space $V=[0,1]^{2}$. If the seller knows only the rankings of the realized valuations, his information is given by the partition $\pi=\{H, L\}$ of $V$ where

$$
\begin{aligned}
& H=\left\{v=\left(v_{1}, v_{2}\right) \in V \mid v_{1} \geq v_{2}\right\} \\
& L=\left\{v=\left(v_{1}, v_{2}\right) \in V \mid v_{1}<v_{2}\right\}
\end{aligned}
$$

As mentioned, each buyer knows his own valuation. The seller sends a public message and the buyers send sealed bids. The buyer with the higher bid gets the object and pays his bid.

\subsection{Messages that Convey the Truth or Lie}

To gain some intuition about the results we first assume that the seller can only report the rankings. Formally, he can either say $h$ or $l$ where $h$ implies $v_{1} \geq v_{2}$ and $l$ implies $v_{1}<v_{2}$, that is, $I(h)=H$ and $I(l)=L$. A (pure) strategy is then a mapping $s:\{H, L\} \rightarrow\{h, l\}$. Assume further that he is restricted to one of the two strategies:

$$
\begin{array}{rll}
\text { truth telling, } s^{t} & : & s^{t}(H)=h, s^{t}(L)=l \\
\text { lying, } s^{l} & : & s^{l}(H)=l, s^{l}(L)=h
\end{array}
$$

With this restriction a seller's mixed strategy is a probability $p(0 \leq p \leq 1)$ that he will transmit the true information (i.e. $p$ is the probability of using $s^{t}$ and $(1-p)$ is the probability of using $\left.s^{l}\right){ }^{8}$

We restrict our attention to equilibria where the buyers use a differentiable, strictly-increasing bid function. With a minor abuse of notation, we shall write $b_{l}^{p}$ for the bid function of the low-signal buyer (buyer 1 when $l$ is announced and buyer 2 when $h$ is announced) when the seller's strategy is $p$. Similarly, we write $b_{h}^{p}$ for the bid function of the high-signal buyer. Thus, an equilibrium consists of $\left(p, b_{l}^{p}, b_{h}^{p}\right)$ where both $b_{l}^{p}$ and $b_{h}^{p}$ are best reply to each other given $p$, and $p$ maximizes the seller's revenue given $\left(b_{l}^{p}, b_{h}^{p}\right)$.

\footnotetext{
${ }^{8}$ One may allow any mixed strategy based on this alphabet, i.e. any probability distribution on the pure strategies $s^{t}, s^{l}$ and the other two strategies of always announcing $h$ or always announcing $l$. We do not do this since it would complicate the proof and obscure the intuitive argument and anyway, this is a special case of stronger result presented in the following subsection.
} 
Lemma $3.1 b_{l}^{p}(1)=b_{h}^{p}(1)$. (That is, at the value of 1, a buyer bids independent of the announced message.)

Proof: Assume by contradiction $b_{l}^{p}(1)>b_{h}^{p}(1)$. This cannot be part of an equilibrium since a low-value buyer with valuation 1 can profit by deviating to a strictly lower bid that is still above $b_{h}^{p}(1)$ (for example, $\left(b_{l}^{p}(1)+b_{h}^{p}(1)\right) / 2$ ); he still wins with certainty (because the bid functions are increasing), but pays less. Similarly, $b_{l}^{p}(1)<b_{h}^{p}(1)$ cannot hold in equilibrium either.

Lemma 3.2 When $p>0.5, b_{l}^{p}(v)>b_{h}^{p}(v)$ for all $v$ in $(0,1)$. When $p<0.5$, $b_{l}^{p}(v)<b_{h}^{p}(v)$ for all $v$ in $(0,1)$.

Proof: The proof is by contradiction. In both cases of $p$, we will show that if a point exists where the opposite relationship of $b_{h}^{p}$ and $b_{l}^{p}$ holds then, the differential equations derived from the equilibrium first-order conditions imply that these functions cannot meet at any value greater than this point, in contradiction to Lemma 3.1.

Fix $p>0.5$ and assume that for some $v(0<v<1), b_{l}^{p}(v)<b_{h}^{p}(v)$. For simplicity of notation, we omit the superscript $p$ and write $b_{l}$ and $b_{h}$ for the bidding functions at equilibrium (at $p$ ) and their inverse functions by $v_{l}$ and $v_{h}$, respectively. The (conditional) expected payoff of a low-signal buyer with value $v$ when bidding $b=b_{l}(v)$ is (note that since $b_{l}(v)<b_{h}(v)$ and $b_{h}$ is increasing, the low-signal buyer with value $v$ will certainty lose if he has in fact the low value):

$$
\frac{(1-p) F(v)}{(1-p) F(v)+p(1-F(v))} \cdot \frac{F\left(v_{h}(b)\right)}{F(v)}(v-b)
$$

The expected payoff of a high-signal buyer with value $v$ when bidding $b=b_{h}(v)$ is:

$$
\left[\frac{p F(v)}{(1-p)(1-F(v))+p F(v)}+\frac{(1-p)(1-F(v))}{(1-p)(1-F(v))+p F(v)} \cdot \frac{F\left(v_{l}(b)\right)-F(v)}{1-F(v)}\right] \cdot(v-b)
$$

The first order conditions that (1) is maximized at $b=b_{l}\left(v_{l}\right)$ and $(2)$ is maximized at $b=b_{h}\left(v_{h}\right)$ are:

$$
\begin{gathered}
v_{l}^{\prime}=\frac{p F\left(v_{h}\right)+(1-p)\left(F\left(v_{l}\right)-F\left(v_{h}\right)\right)}{\left(v_{h}-b\right)(1-p) F^{\prime}\left(v_{l}\right)} \\
v_{h}^{\prime}=\frac{F\left(v_{h}\right)}{\left(v_{l}-b\right) F^{\prime}\left(v_{h}\right)}
\end{gathered}
$$

These equations would be valid as long as $b_{l}(v)<b_{h}(v)$ or until the two bid functions meet. From Lemma 3.1, we know that the bid functions must meet at a point greater than $v$. Let $\hat{v}>v$ be the meeting point closest to $v$. At such a 
point, the $b_{l}$ would meet $b_{h}$ from below, which implies $b_{h}^{\prime}(\hat{v}) \leq b_{l}^{\prime}(\hat{v})$. But from the differential equations,

$$
\lim _{v \rightarrow \hat{v}} \frac{b_{h}^{\prime}}{b_{l}^{\prime}}=\frac{v_{l}^{\prime}}{v_{h}^{\prime}}=\frac{p}{1-p}
$$

Since $p>.5$, this limit is strictly greater than 1 . Thus, $b_{h}^{\prime}(\hat{v})>b_{l}^{\prime}(\hat{v})$ which contradicts $b_{h}^{\prime}(\hat{v}) \leq b_{l}^{\prime}(\hat{v})$ and implies that they cannot meet at a point greater than $v$ violating Lemma 3.1. Similarly, when $p<.5$, there is also a contradiction if $b_{l}(v)>b_{h}(v)$.

Proposition 3.1 In all equilibria of this game, the seller uses strategy $p=.5$.

Proof: Let $v_{H}$ be the (true) high value and $v_{L}$ be the low value. The seller's expected revenue is

$$
p \cdot \max \left\{b_{h}^{p}\left(v_{H}\right), b_{l}^{p}\left(v_{L}\right)\right\}+(1-p) \cdot \max \left\{b_{h}^{p}\left(v_{L}\right), b_{l}^{p}\left(v_{H}\right)\right\}
$$

First, assume that $p>$.5. Since $b_{l}^{p}(v)>b_{h}^{p}(v)$ for all $v \in(0,1)$ by Lemma 3.2 , we have almost surely that $b_{l}^{p}\left(v_{H}\right)>b_{h}^{p}\left(v_{H}\right)$ and $b_{l}^{p}\left(v_{H}\right)>b_{l}^{p}\left(v_{L}\right)$ and hence deviating to $p=0$ (always lying) will be profitable. Thus, any $p>.5$ cannot be part of an equilibrium. Likewise, any $p<.5$ cannot be part of an equilibrium as well. Therefore, the only possible equilibrium strategy for the seller is $p=.5$.

Note that a strategy of $p=.5$ is equivalent to sending no information at all. This establishes our assertion that by publicly communicating the rankings with no commitment power, the seller cannot increase his revenue.

\subsection{Multiple Signals}

We now strengthen the results of the previous section by showing that when the seller knows the rankings and has no commitment power, he cannot gain from his information even with a richer set of signals.

Assume that the seller has the ability to send any message from an alphabet $A=\left\{a_{1}, a_{2}, \ldots\right\}$. Given a randomized strategy (either mixed or behavioral), ${ }^{9}$ let $h_{i}=P\left(a_{i} \mid H\right)$ and $l_{i}=P\left(a_{i} \mid L\right)$ (that is, $h_{i}$ is the probability that the seller sends $a_{i}$ when he has information $H$ and similarly for $\left.l_{i}\right)$.

Let $P\left(L \mid a_{i}, v_{j}\right)$ be the probability of the event $L\left(v_{1}<v_{2}\right)$ given signal $a_{i}$ and given the value of buyer $j$ is $v_{j}$. (Note that $P\left(a_{i} \mid L, v_{i}\right)=P\left(a_{i} \mid L\right)=l_{i}$

\footnotetext{
${ }^{9}$ By Kuhn's Theorem, there is no loss of generality in considering only strategies in $\{s$ : $\{H, L\} \rightarrow \triangle(A)\}$ (behavioral strategies) since the seller in this game has perfect recall (since he has only one move).
} 
and $P\left(a_{i} \mid H, v_{i}\right)=P\left(a_{i} \mid H\right)=h_{i}$, since the seller's strategy is a function of his information - $H$ or $L$ - only.) By Bayes' rule, we have:

$$
\begin{aligned}
P\left(L \mid a_{i}, v_{2}\right) & =P\left(v_{1}<v_{2} \mid a_{i}, v_{2}\right)=\frac{F\left(v_{2}\right) l_{i}}{F\left(v_{2}\right) l_{i}+\left(1-F\left(v_{2}\right)\right) h_{i}} \\
P\left(H \mid a_{i}, v_{2}\right) & =1-P\left(L \mid a_{i}, v_{2}\right)=\frac{\left(1-F\left(v_{2}\right)\right) h_{i}}{F\left(v_{2}\right) l_{i}+\left(1-F\left(v_{2}\right)\right) h_{i}}
\end{aligned}
$$

First notice that $\sum_{i} h_{i}=\sum_{i} l_{i}=1$. Hence, there exists a $j$ such that $h_{j}>l_{j}$ if and only if there exists a $k$ such that $h_{k}<l_{k}$.

Denote by $b_{1}^{i}$ and $b_{2}^{i}$ the equilibrium bidding functions of the two buyers given message $a_{i}$, and assume there are strictly increasing and differentiable. Denote by $v_{1}^{i}$ and $v_{2}^{i}$ the corresponding inverse functions.

The following lemma confirms the intuition that in equilibrium, a buyer who is more likely to have the lower valuation (given the signal) bids more aggressively than the other buyer, who is more likely to have the higher valuation.

Lemma 3.3 If $h_{k}<l_{k}$, then $b_{1}^{k}(v)>b_{2}^{k}(v)$ for all $v$ in $(0,1)$.

If $h_{j}>l_{j}$, then $b_{1}^{j}(v)<b_{2}^{j}(v)$ for all $v$ in $(0,1)$.

Proof: We prove the first statement. The proof is similar to that of Lemma 3.2.

If the statement is not true, then for some $k$, we have $h_{k}<l_{k}$ and some neighborhood in $(0,1)$ where $b_{2}^{k}(v)>b_{1}^{k}(v)$. When $v_{1}$ is in such a region and buyer 1 bids $x$ in the neighborhood of $b_{1}^{k}\left(v_{1}\right)$, his expected payoff is equal to

$$
P\left(H \mid a_{k}, v_{1}\right) \cdot \frac{F\left(v_{2}^{k}(x)\right)}{F\left(v_{1}\right)}\left(v_{1}-x\right)
$$

Also, when $v_{2}$ is in such a region and buyer 2 bids $x$ in the neighborhood of $b_{2}^{k}\left(v_{2}\right)$, his expected payoff is equal to

$$
P\left(L \mid a_{k}, v_{2}\right)\left(v_{2}-x\right)+P\left(H \mid a_{k}, v_{2}\right) \cdot\left(\frac{F\left(v_{1}^{k}(x)\right)-F\left(v_{2}\right)}{1-F\left(v_{2}\right)}\right)\left(v_{2}-x\right)
$$

We look at the first-order conditions of the above expected payoffs since each bid must maximize expected payoff and each global maximum must also be a local maximum.

$$
\begin{gathered}
v_{1}^{k^{\prime}}(x)=\frac{\frac{P\left(L \mid a_{k}, v_{2}\right)}{P\left(H \mid a_{k}, v_{2}\right)}\left(1-F\left(v_{2}\right)\right)+F\left(v_{1}\right)-F\left(v_{2}\right)}{F^{\prime}\left(v_{1}\right)\left(v_{2}-x\right)} \\
v_{2}^{k^{\prime}}(x)=\frac{F\left(v_{2}\right)}{\left(v_{1}-x\right) F^{\prime}\left(v_{2}\right)}
\end{gathered}
$$

These equations hold when both $v_{1}$ and $v_{2}$ are in the region where $b_{2}^{k}(v)>$ $b_{1}^{k}(v)$. Let $\hat{v}$ be the smallest value such that $\hat{v}>v$ and $b_{1}^{k}(\hat{v})=b_{2}^{k}(\hat{v})=\hat{x}$. We have (using (3) and (4)): 


$$
\lim _{x \rightarrow \hat{x}} \frac{v_{1}^{k^{\prime}}}{v_{2}^{k^{\prime}}}=\lim _{v_{1}, v_{2} \rightarrow \hat{v}} \frac{P\left(L \mid a_{k}, v_{2}\right)}{P\left(H \mid a_{k}, v_{2}\right)} \cdot \frac{1-F\left(v_{2}\right)}{F\left(v_{2}\right)}=\frac{l_{k}}{h_{k}}>1
$$

Thus, we obtain that when $b_{1}^{k}(v)>b_{2}^{k}(v)$, at the next meeting point one has $b_{1}^{k^{\prime}}(\hat{v})>b_{2}^{k^{\prime}}(\hat{v})$. Since this is impossible when $v<\hat{v} \leq 1$, we conclude that there is no such $\hat{v}$, in contradiction to the fact that $b_{1}^{k}(1)=b_{2}^{k}(1)$ (a straightforward generalization of Lemma 3.1 to the case of any number of signals).

In a similar way it is shown that if $h_{j}>l_{j}$ then $b_{2}^{j}(v)>b_{1}^{j}(v)$ for all $v$ in $(0,1)$.

Let $\pi\left(a_{i} \mid H\right)$ be the expected seller's revenue when signal $a_{i}$ is sent and buyer 1 has the highest valuation and $\pi\left(a_{i} \mid L\right)$ be the expected seller's revenue when signal $a_{i}$ is sent and buyer 1 has the lowest valuation. Note that

$$
\begin{aligned}
& \pi\left(a_{j} \mid L\right)=\int_{v_{1}<v_{2}} \pi\left(a_{i} \mid v\right) \cdot f\left(v \mid v_{1}<v_{2}\right) d v \\
& \pi\left(a_{j} \mid H\right)=\int_{v_{1} \geq v_{2}} \pi\left(a_{i} \mid v\right) \cdot f\left(v \mid v_{1} \geq v_{2}\right) d v
\end{aligned}
$$

Lemma 3.4 If $h_{j}>l_{j}$, then $\pi\left(a_{j} \mid L\right)>\pi\left(a_{j} \mid H\right)$ and if $h_{k}<l_{k}$, then $\pi\left(a_{k} \mid H\right)>$ $\pi\left(a_{k} \mid L\right)$.

Proof: Let us show that $\pi\left(a_{j} \mid L\right)>\pi\left(a_{j} \mid H\right)$. First notice that for any values $0<\underline{v}<\bar{v}<1, f\left((\underline{v}, \bar{v}) \mid v_{1}<v_{2}\right)=f\left((\bar{v}, \underline{v}) \mid v_{1} \geq v_{2}\right)$ (we assume there is a symmetric continuous distribution of values). Now notice that $\pi\left(a_{j} \mid(\underline{v}, \bar{v})\right)=b_{2}^{j}(\bar{v})$ and $\pi\left(a_{j} \mid(\bar{v}, \underline{v})\right)=\max \left\{b_{1}^{j}(\bar{v}), b_{2}^{j}(\underline{v})\right\}$. However, $b_{2}^{j}(\bar{v})>\max \left\{b_{1}^{j}(\bar{v}), b_{2}^{j}(\underline{v})\right\}$, which implies $\pi\left(a_{j} \mid(\underline{v}, \bar{v})\right)>\pi\left(a_{j} \mid(\bar{v}, \underline{v})\right)$. Thus, by comparing the integrals pointwise, we find that $\pi\left(a_{j} \mid L\right)>\pi\left(a_{j} \mid H\right)$. In a similar manner, we can show that $\pi\left(a_{k} \mid H\right)>\pi\left(a_{k} \mid L\right)$.

Proposition 3.2 In any equilibrium, (i) $h_{i}=l_{i}$ for all $i$ (i.e., no information regarding the rankings is conveyed by the seller's signaling). (ii) The seller's expected revenue is the same as in the standard model with no signaling.

Proof: If (i) does not hold, then there are $j$ and $k$ such that $h_{j}>l_{j}$ and $h_{k}<l_{k}$. This implies that $h_{j}=P\left(a_{j} \mid H\right)>0$ and $l_{k}=P\left(a_{k} \mid L\right)>0$. For this to be part of an equilibrium, the seller must have no incentive to always send $a_{k}$ instead of $a_{j}$ in state $H$ and no incentive to always send $a_{k}$ instead of $a_{j}$ in state $L$, that is: $\pi\left(a_{j} \mid H\right) \geq \pi\left(a_{k} \mid H\right)$ and $\pi\left(a_{k} \mid L\right) \geq \pi\left(a_{j} \mid L\right)$. By the first part of Lemma 3.4, this implies $\pi\left(a_{k} \mid L\right) \geq \pi\left(a_{j} \mid L\right)>\pi\left(a_{j} \mid H\right) \geq \pi\left(a_{k} \mid H\right)$, which contradicts the second part of Lemma $3.4\left(\pi\left(a_{k} \mid L\right)<\pi\left(a_{k} \mid H\right)\right)$. Thus, for all messages $h_{i}=l_{i}$.

While we study only public messages, the results of no commitment also hold for private messages (we omit the proof). This agrees with Farrell and Gibbons [1989] who study the difference between public and private messages in a 
game with no commitment power and two receivers. Under symmetry, their results imply that any equilibrium with meaningful private messages also exists with meaningful public messages. Thus, if there is no equilibrium with meaningful public messages, there won't be an equilibrium with meaningful private messages.

\section{Partial Commitment}

Consider a situation in which the seller knows the valuations and can send as a public message the valuations, the rankings, or a white signal $\phi$ (equivalent to sending no signal). ${ }^{10}$ Formally, the seller's information is

$$
\pi=\{\{v\} \mid v \in V\}
$$

his alphabet is

$$
A=\{\phi,(l, h),(h, l), V\}
$$

and his set of signaling strategies, as mentioned before, is

$$
S=\{s: V \rightarrow A\}
$$

Under partial (truthful) commitment, this strategy would be limited to signals which are truthful. We can write the appropriate interpretation function as $I((h, l))=\left\{v \mid v \in V\right.$ and $\left.v_{1} \geq v_{2}\right\}, I((l, h))=\left\{v \mid v \in V\right.$ and $\left.v_{1}<v_{2}\right\}$, $I\left(\left(v_{1}, v_{2}\right)\right)=\left\{\left(v_{1}, v_{2}\right)\right\}$, and $I(\phi)=V$.

With partial commitment, the seller must choose his pure strategy from the truthful strategy set:

$$
S^{t}=\{s \in S \mid v \in I(s(v)), \forall v \in V\}
$$

Which in this case is equivalent to:

$$
S^{t}=\left\{\begin{array}{l|l}
s \in S & \begin{array}{l}
s(v) \in V \Rightarrow s(v)=v \\
s(v)=(l, h) \Rightarrow v_{1}<v_{2} \\
s(v)=(h, l) \Rightarrow v_{2} \leq v_{1}
\end{array}
\end{array}\right\}
$$

That is, the seller could send $(h, l)$ only when $v_{1} \geq v_{2}$, the rankings of $(l, h)$ only when $v_{1}<v_{2}$, the values $\left(v_{1}, v_{2}\right)$ only when these are the true values, or

\footnotetext{
${ }^{10}$ We should note that under such information conditions either a mechanism of making a take-it-or-leave-it offer of $v_{h}$ to the high-value buyer or a first-price auction with a reserve price set to $v_{h}$ will give the seller all the possible surplus. In spite of this, our interest in a first-price auction without a reservation price is more than just academic. In addition to the several reasons mentioned in the introduction for a seller being restricted in his ability to redesign the auction, the lack of a possible reservation price could be due to a seller being forced to sell. A buyer knowing this would not believe any set reservation price (or take-it-or-leave-it offer).
} 
$\phi$ in any case. Thus, for any particular set of values $\left(v_{1}, v_{2}\right)$, there are only three truthful signals. Let us call these options $\phi, r$, and $v$, where $r$ (stands for rankings) is the appropriate choice of $(h, l)$ or $(l, h)$. Therefore, an equivalent choice for the seller is the choice of 'pressing one of three buttons' labeled $\phi, r$ and $v$.

In examining the equilibria of this game, we make the assumption:

Assumption 4.1 A buyer never bids above his value.

As opposed to full (truthful) commitment, any equilibrium strategy of the seller must be profit maximizing given his information. That is, given any pair of valuations $v_{1}, v_{2}$ if in equilibrium $r$ is sent, it must generate revenue at least as high as if $v$ or $\phi$ were sent. Likewise for $v$ and $\phi$.

Using this property, we can now characterize the set of equilibria by the following lemmatta (the first Lemma is well known but for completeness we provide its proof in the Appendix). Throughout this section, we consider the possibility of mixed strategies on the part of the buyers. That is, the equilibrium strategies of the buyers, given $v$ and the signal sent, consist of distributions on $b_{l}$ and $b_{h}$.

Lemma 4.1 In any equilibrium, if $v$ is sent, then if $v_{l}<v_{h}$, the winning bid will be at the lowest valuation and won by the high-value buyer: $b_{h}=v_{l}$ and $P\left(b_{l}<v_{h}\right)=1$. If $v_{l}=v_{h}=v$ then both buyers bid $v$ with probability one (and hence the winning bid will, again be $v)$.

The proof is given in the Appendix. $\square$.

Lemma 4.2 In any equilibrium of the game with the seller, the expected winning bid will be weakly greater than the lower valuation: $E\left[\max \left\{b_{l}, b_{h}\right\} \mid v_{l}, v_{h}\right] \geq v_{l}$.

Proof: If the expected winning bid were strictly lower than the lower valuation, then the seller could profitably deviate by sending $v$ and getting the lowest value by Lemma 4.1 .

For $c \geq 0$ let $D_{c}=\{(x, x) \mid c<x \leq 1\}$ (i.e. the left-open $c$-upper part of the diagonal of $V$ ), and for $\epsilon>0$ let $D_{c}^{\epsilon}$ be the $\epsilon$-neighborhood in $V$ (in the maximum norm) of $D_{c}$. Denote by $V(\phi)$ the subset of $V$ in which the seller sends the signal $\phi$ in equilibrium.

Lemma 4.3 For any $c>0$ there is an $\epsilon>0$ such that $D_{c}^{\epsilon}$ is disjoint from $V(\phi)$.

Proof: Notice first that this Lemma implies that $V(\phi)$ is disjoint from $D_{0}$ (i.e. that $V(\phi)$ cannot contain a point on the diagonal except possibly $(0,0))$. To gain some intuition on the main argument, let us first prove this fact separately. 
Assume, by contradiction, that $\phi$ is sent by the seller at a point $v_{1}=v_{2}=c>$ 0. By Lemma 4.2 and Assumption 4.1, the winning bid must be equal to $c$. This can only happen if both buyers bid $c$ with certainty. (If neither buyer is bidding $c$ with certainty, then, with positive probability, the winning bid would be lower than $c$. If only one buyer bids $c$ with certainty, he would have incentive to lower his bid.) In particular, buyer 2 is bidding $c$ with certainty. Since the seller can send $\phi$ at any time when $v_{1}<v_{2}=c$, the expected winning bid must be weakly greater than $c$ for all $v_{1}<v_{2}=c$. This can only occur if $b_{2}=c$ for all values $v_{1}<v_{2}=c$. However, this is cannot be an equilibrium strategy, since buyer 2 has incentive to deviate to bidding, say, $c / 2$ and expect a positive profit.

Returning now to the proof of the Lemma, assume that $v=\left(v_{1}, v_{2}\right) \in V(\phi)$ and $v$ is also in $D_{c}^{\epsilon}$ for $c>0$. Assume that $v_{1} \leq v_{2}$. This means that $\phi$ is sent while:

$$
c-\epsilon \leq v_{1} \leq v_{2} \leq v_{1}+\epsilon
$$

We shall show that for $\epsilon$ small enough, this leads to a contradiction. For a constant $k>0$ (to be specified later) let $q_{1}=P\left(b_{1} \geq v_{1}-k \epsilon\right)$ and $q_{2}=P\left(b_{2} \geq v_{1}-k \epsilon\right)$.

The expected selling price is then at most (using Assumption 4.1 and (5)):

$$
q_{2}\left(v_{1}+\epsilon\right)+\left(1-q_{2}\right)\left(q_{1} v_{1}+\left(1-q_{1}\right)\left(v_{1}-k \epsilon\right)\right)=v_{1}+\epsilon\left(1-k\left(1-q_{1}\right)\left(1-q_{2}\right)\right)
$$

Since, by Lemma 4.2 , this is at least $v_{1}$, we have

$$
1-k\left(1-q_{1}\right)\left(1-q_{2}\right) \geq 0
$$

Letting $z=\max \left\{q_{1}, q_{2}\right\}$ and $z(k)=1-\sqrt{1 / k}$, this implies $z \geq z(k)$. We can deduce that at least one buyer, say $i$, bids so that $P\left(b_{i} \geq v_{1}-k \epsilon\right) \geq z(k)$ and hence the expected selling price is at least $z(k)\left(v_{1}-k \epsilon\right)$, and consequently, the expected payoff of buyer $i$ is at most:

$$
\begin{aligned}
v_{i}-z(k)\left(v_{1}-k \epsilon\right) & \leq v_{1}+\epsilon-z(k)\left(v_{1}-k \epsilon\right) \\
& =v_{1} \cdot(1-z(k))+\epsilon \cdot(1+k \cdot z(k))
\end{aligned}
$$

Choose large enough $k$ such that $1-z(k)<\frac{1}{8} \frac{c}{2} F\left(\frac{c}{2}\right)$, then choose $\epsilon_{1}$ such that for $\epsilon<\epsilon_{1}, \epsilon(1+k z(k))<\frac{1}{8}\left(\frac{c}{2}\right) F\left(\frac{c}{2}\right)$. We conclude that if $\epsilon<\epsilon_{1}$, and $\left(v_{1}, v_{2}\right) \in V(\phi) \cap D_{c}^{\epsilon}$, then there is a buyer $i$ with equilibrium expected payoff less than (by (6)) $\frac{1}{4} \cdot \frac{c}{2} F\left(\frac{c}{2}\right)$.

On the other hand, by ignoring the seller's message and bidding $b_{i}=v_{i} / 2$, buyer $i$ guarantees expected payoff of at least $\left(\frac{v_{i}}{2}\right) F\left(\frac{v_{i}}{2}\right) \geq \frac{c-\epsilon}{2} F\left(\frac{c-\epsilon}{2}\right)$, which can be made larger than $\frac{1}{2} \frac{c}{2} F\left(\frac{c}{2}\right)$ for $\epsilon<\epsilon_{2}$. For $\epsilon<\min \left\{\epsilon_{1}, \epsilon_{2}\right\}$ this yields a profitable deviation from the equilibrium strategy; a contradiction which completes the proof. 
Definition: Given a strategy $\sigma \in \Sigma^{t}$ of the seller and buyer $i$ with value $v_{i}$, we say that $v_{-i}$ is consistent (for $i$ ) with signal $a$ if $a \in \sigma\left(v_{i}, v_{-i}\right.$ ) (recall that $\sigma(v)=\{s(v) \mid s \in \operatorname{Supp}(\sigma)\})$.

Definition: We say that a buyer $i$ makes an h-bid if when he has a value $v_{i}$ and receives a signal $a$, he bids

$$
b_{i}\left(v_{i}, a\right)=\sup \left\{v_{-i} \mid v_{-i} \leq v_{i} \text { and } v_{-i} \text { is consistent with the signal } a\right\}
$$

whenever the set over which the sup is taken is not empty.

That is, given the signal sent and beliefs about the seller's strategy, a buyer can form beliefs about the other buyer's possible valuations; he makes an h-bid (based on his beliefs) if he bids the sup of all other buyer's possible values that are lower than his own valuation based one's beliefs, if, indeed, there are such values.

Lemma 4.4 In any equilibrium, if a high-value buyer knows (with certainty) he has the high value, he makes an h-bid.

Proof: When the signal sent is $v$, this follows from Lemma 4.1. When the signal sent is $r$ or $\phi$, from Lemma 4.2, we know that the expected winning bid must be at least $v_{l}$. Denoting by $\bar{v}_{l}$, the sup of the $v_{l}$ values compatible with the respective signal in equilibrium, we must also have $E\left[\max \left\{b_{h}, b_{l}\right\} \mid v_{l}, v_{h}\right] \geq \bar{v}_{l}$. Since buyer $H$ knows he has the highest valuation, he also knows that buyer $L$ will bid at most $\bar{v}_{l}$. Therefore, in equilibrium, he will never bid more than $\bar{v}_{l}$. Since buyer $L$ also never bids more than $\bar{v}_{l}$, the winning bid when $v_{l}=\bar{v}_{l}$ must equal $\bar{v}_{l}$. This can only occur if at least one of the two buyers is bidding $\bar{v}_{l}$ with certainty. If $P\left(b_{h}=\bar{v}_{l}\right)<1$, any buyer $L$, with value close enough to $\bar{v}_{l}$, would have incentive to bid below $\bar{v}_{l}$. Therefore, $b_{h}=\bar{v}_{l}$.

Lemma 4.5 In any equilibrium, if a buyer $i$ with value $v_{i}$ knows that whenever the other buyer $j$ has a value $v_{j}>v_{i}$ he makes an h-bid, then buyer $i$ also makes an h-bid.

Proof: This is only different from Lemma 4.4 when the signal sent is $\phi$. A buyer with value $v_{i}$ who bids $b$ expects a payoff of $g_{h}(b) \cdot P\left(v_{i}=v_{h}\right)+g_{l}(b) \cdot P\left(v_{i}=v_{l}\right)$, where $g_{h}(b)$ is the conditional expected profit from bidding $b$ given that $v_{i}=v_{h}$ and likewise for $g_{l}(b)$. Since the other buyer $j$ makes an h-bid, buyer $i$ cannot win when his value $v_{i}$ is the low value. Thus, the equilibrium bid $b^{*}$ is such that $g_{l}\left(b^{*}\right)=0$, and thus $b^{*}$ maximizes $g_{h}(b)$, which leads us to $b^{*}=\bar{v}_{l}$ by the argument in Lemma 4.4.

Lemma 4.6 In equilibrium, each buyer makes an h-bid. 
Proof: By Lemma 4.4, this is true when the signals are $v$ or $r$ are sent (since they are truthful). So consider $v=\left(v_{1}, v_{2}\right)$ in $V(\phi)$. We know by Lemma 4.3 that $V(\phi)$ does not intersect the diagonal $D_{0}$. If $V(\phi)$ lies on one side of $D_{0}$, then $\phi$ reveals the rankings and the result follows from Lemma 4.4. Otherwise, if buyer 1 does not make an h-bid at $v=\left(v_{1}, v_{2}\right) \in V(\phi)$, then, by Lemma 4.5, there must be $v_{2}^{1}>v_{1}$ such that $\left(v_{1}, v_{2}^{1}\right) \in V(\phi)$ and buyer 2 does not make an h-bid at $\left(v_{1}, v_{2}^{1}\right)$. Applying Lemma 4.5 again, there must be $v_{1}^{1}>v_{2}^{1}$ such that $\left(v_{1}^{1}, v_{2}^{1}\right) \in V(\phi)$ and buyer 1 does not make an h-bid at $\left(v_{1}^{1}, v_{2}^{1}\right)$. Using repeatedly this argument, we conclude that there is a chain of points:

$$
\left(v_{1}, v_{2}\right),\left(v_{1}, v_{2}^{1}\right),\left(v_{1}^{1}, v_{2}^{1}\right),\left(v_{1}^{1}, v_{2}^{2}\right), \ldots,\left(v_{1}^{t}, v_{2}^{t}\right), \ldots
$$

in $V(\phi)$ such that

$$
v_{1}<v_{2}^{1}<v_{1}^{1}<v_{2}^{2}<v_{1}^{2}<\ldots<v_{2}^{t}<v_{1}^{t}<v_{2}^{t+1}<\ldots
$$

By Lemma 4.3 (applied for $\epsilon>0$ corresponding to $c=v_{1}>0$ ) we have $v_{1}^{t+1}-v_{1}^{t}>\epsilon$ for all $t$. This implies $v^{t} \stackrel{t \rightarrow \infty}{\longrightarrow} \infty$, a contradiction which concludes the proof.

Lemma 4.7 The auction is efficient: with probability one, the high-value buyer will win the auction.

Proof: Assume that in equilibrium $L$ wins the auction with positive probability. This must occur when $L$ bids his value, $b_{l}=v_{l}$ (since by Lemma $4.6, b_{h} \geq v_{l}$ ).

We already know (by Lemma 4.2) that when $v$ is sent, the outcome is efficient, therefore any inefficiency must occur when $r$ or $\phi$ is sent. When $L$ wins, he bids his value and thus must know for sure that he has the low value even if $\phi$ is sent. If this were not the case, by Assumption 4.1, he can generate positive expected profits by bidding less than his value since he will have the high value and win the auction with positive probability.

Since $L$ wins with positive probability, there is a region $W \subset V$ where this happens and $P(W)>0$. Without loss of generality, we may assume that $W$ is a rectangle: $W=\left[\underline{v}_{l}, \bar{v}_{l}\right] \times\left[\underline{v}_{h}, \bar{v}_{h}\right]$ (just take a rectangular neighborhood in any such $W)$. So for all $v \in W$ and $v_{h} \in\left[\underline{v}_{h}, \bar{v}_{h}\right], H$ loses with positive probability against $L$, who is bidding his value, hence $P\left(b_{h} \leq \underline{v}_{l}\right)>0$. However, in this region, $L$ with value $\bar{v}_{l}$ can profitably deviate to bid $\left(\underline{v}_{l}+\bar{v}_{l}\right) / 2$, a contradiction.

Lemma 4.8 In any equilibrium, the seller receiving information $\left(v_{l}, v_{h}\right)$ would not send $v$ if there is $a\left(v_{l}^{\prime}, v_{h}\right)$ such that $v_{l}<v_{l}^{\prime}<v_{h}$ where $r$ or $\phi$ is sent.

Proof: Suppose that there is a $v=\left(v_{l}, v_{h}\right)$ where $v$ is sent and there is a $\left(v_{l}^{\prime}, v_{h}\right)$, with $v_{l}<v_{l}^{\prime}<v_{h}$, where $r$ or $\phi$ is sent. The seller's revenue at $v=\left(v_{l}, v_{h}\right)$ will be $v_{l}$, while his revenue at $\left(v_{l}^{\prime}, v_{h}\right)$ will be at least $v_{l}^{\prime}$ (by Lemma 4.2). Since $v_{l}^{\prime}>v_{l}$, the seller would have incentive to send $r$ or $\phi$ at $\left(v_{l}, v_{h}\right)$ instead of $v$ and receive at least $v_{l}^{\prime}$. Thus, this could not be an equilibrium. 
Lemma 4.9 In any equilibrium, for $s \in\{r, \phi\}$, if $s \in \sigma\left(v_{l}, v_{h}\right)$, then there exists $\bar{v}_{l} \geq v_{l}$ such that $\bar{v}_{l} \in a\left(v_{h}\right)$ and $s \in \sigma\left(\bar{v}_{l}, v_{h}\right)$, where $a\left(v_{h}\right)=\left\{x \mid\left(v_{h}-x\right) F(x) \geq\right.$ $\left(v_{h}-b\right) F(b)$ for all $\left.b \in[0, x]\right\}$. Furthermore, if $r \in \sigma\left(v_{l}, v_{h}\right)$ and $\phi \in \sigma\left(v_{l}, v_{h}\right)$, then there exists $\bar{v}_{l} \geq v_{l}$ such that $\bar{v}_{l} \in a\left(v_{h}\right), r \in \sigma\left(\bar{v}_{l}, v_{h}\right)$ and $\phi \in \sigma\left(\bar{v}_{l}, v_{h}\right)$.

Proof: Let $V\left(v_{h}, \phi\right)=\left\{v_{l} \mid \phi \in \sigma\left(v_{l}, v_{h}\right)\right\}$ and $V\left(v_{h}, r\right)=\left\{v_{l} \mid r \in \sigma\left(v_{l}, v_{h}\right)\right\}$. Also let $\bar{v}_{l, \phi}\left(v_{h}\right)=\sup \left\{v_{l} \mid v_{l} \in V\left(v_{h}, \phi\right)\right\}$ and $\bar{v}_{l, r}\left(v_{h}\right)=\sup \left\{v_{l} \mid v_{l} \in V\left(v_{h}, r\right)\right\}$.

By Lemma 4.6 and Lemma 4.7, if in equilibrium $\phi \in \sigma\left(\bar{v}_{l, \phi}\left(v_{h}\right), v_{h}\right)$, then $H$ bids $\bar{v}_{l, \phi}\left(v_{h}\right)$ and wins, while if $r \in \sigma\left(\bar{v}_{l, r}\left(v_{h}\right), v_{h}\right)$, then $H$ bids $\bar{v}_{l, r}\left(v_{h}\right)$ and wins. It follows that $\bar{v}_{l, \phi}\left(v_{h}\right)=\bar{v}_{l, r}\left(v_{h}\right)=: \bar{v}_{l}\left(v_{h}\right)$ (since otherwise the seller would have a profitable deviation to either send $r$ at $\left(\bar{v}_{l, \phi}\left(v_{h}\right), v_{h}\right)$ or send $\phi$ at $\left.\left(\bar{v}_{l, r}\left(v_{h}\right), v_{h}\right)\right)$.

By Lemma 4.8, $V\left(v_{h}, \phi\right) \cup V\left(v_{h}, r\right)=\left[0, \bar{v}_{l}\left(v_{h}\right)\right]$. Since at $\left(\bar{v}_{l}\left(v_{h}\right), v_{h}\right)$, when $\phi$ or $r$ is sent, buyer $H$ 's equilibrium strategy is to bid $\bar{v}_{l}$ and then win, we have

$$
\begin{aligned}
& v_{h}-\bar{v}_{l} \geq P(b \mid r)\left(v_{h}-b\right) \text { for all } b \in\left[0, \bar{v}_{l}\right] \\
& v_{h}-\bar{v}_{l} \geq P(b \mid \phi)\left(v_{h}-b\right) \text { for all } b \in\left[0, \bar{v}_{l}\right]
\end{aligned}
$$

where $P(b \mid s)$ is the probability that $H$ will win the auction with bid $b$ given signal $s$ is sent.

Let $G_{r}(x)=P\left(v_{l} \leq x \mid v_{h}, r\right)$ and $G_{\phi}(x)=P\left(v_{l} \leq x \mid v_{h}, \phi\right)$. By Assumption 4.1 (buyers do not bid higher than their valuations), we know that for all $b \leq \bar{v}_{l}$,

$$
P(b \mid r) \geq \frac{G_{r}(b)}{G_{r}\left(\bar{v}_{l}\right)} \text { and } P(b \mid \phi) \geq \frac{G_{\phi}(b)}{G_{\phi}\left(\bar{v}_{l}\right)}
$$

From (7), (8) and (9), we obtain

$$
\begin{aligned}
& G_{r}\left(\bar{v}_{l}\right)\left(v_{h}-\bar{v}_{l}\right) \geq G_{r}(b)\left(v_{h}-b\right) \\
& G_{\phi}\left(\bar{v}_{l}\right)\left(v_{h}-\bar{v}_{l}\right) \geq G_{\phi}(b)\left(v_{h}-b\right)
\end{aligned}
$$

Multiplying the first inequality by $P\left(r \mid v_{h}\right)$, the second by $P\left(\phi \mid v_{h}\right)$ and adding yields:

$$
\left(G_{r}\left(\bar{v}_{l}\right) P\left(r \mid v_{h}\right)+G_{\phi}\left(\bar{v}_{l}\right) P\left(\phi \mid v_{h}\right)\right)\left(v_{h}-\bar{v}_{l}\right) \geq\left(G_{r}(b) P\left(r \mid v_{h}\right)+G_{\phi}(b) P\left(\phi \mid v_{h}\right)\right)\left(v_{h}-b\right)
$$

Thus,

$$
F\left(\bar{v}_{l}\right)\left(v_{h}-\bar{v}_{l}\right) \geq F(b)\left(v_{h}-b\right) \text { for all } b \in\left[0, \bar{v}_{l}\right]
$$

This concludes the proof.

Corollary 4.1 When the distribution function is uniform, the limit for $\bar{v}_{l}\left(v_{h}\right)$ is $v_{h} / 2$. 
Proof: For the uniform distribution $F(x)=x$, the resulting function $b \cdot\left(v_{h}-b\right)$ attains its maximum at $v_{h} / 2$. The result then follows by Lemma 4.9.

We are now in the position to characterize the equilibria in the partial truthful commitment game.

Proposition 4.1 For any functions $f_{1}$ and $f_{2}$ satisfying $f_{1}(v) \in a(v)$ and $f_{2}(v) \in$ $a(v)$ for all $v \in[0,1]$, where $a(v)$ is defined in Lemma 4.9, the following is an equilibrium in the game $\Gamma_{p t}$ :

- Given his information $v=\left(v_{1}, v_{2}\right)$ the seller sends $v$ if $v_{1} \geq v_{2} \geq f_{1}\left(v_{1}\right)$ or $v_{2} \geq v_{1} \geq f_{2}\left(v_{2}\right)$, otherwise he sends $r$. (Note that the signal $\phi$ is never used in this equilibrium.)

- When $v$ is announced, the buyers play the usual full information equilibrium (Lemma 4.1).

- When $r$ is announced, buyer $i$ with the high valuation bids $f_{i}\left(v_{i}\right)$ and the buyer with the lower valuation bids his value.

- The auction is won always by the high valuation buyer $i$ who pays $v_{j}(j \neq i)$ when $v$ is announced and $f_{i}\left(v_{i}\right)$ otherwise.

Proof: The following strategies and beliefs for the buyers supports such an equilibrium. When $v$ is sent, buyers behave as dictated by Lemma 4.1. When $r$ is sent, buyer $i$ bids his value when low and $f_{i}\left(v_{i}\right)$ when high. This is optimal for buyer $i$ since when low $f_{j}\left(v_{j}\right) \geq v_{i}$ and when high $\left(v_{i}-f_{i}\left(v_{i}\right)\right) \geq\left(F(x) / F\left(f_{i}\left(v_{i}\right)\right)\right)\left(v_{i}-x\right)$ for all $x<f_{i}\left(v_{i}\right)$ (by definition of $a(v)$ ). Off equilibrium beliefs and behavior are as follows. When $\phi$ is sent, buyer $i$ believes that $v_{j} \leq f_{i}\left(v_{i}\right)$ and bids $f_{i}\left(v_{i}\right)$ (the same beliefs and behavior if he receives a signal that he is high). If $r$ is sent when $v_{j}>v_{i}>f_{j}\left(v_{j}\right)$, buyer $j$ does not know that $v_{i} \geq f_{j}\left(v_{j}\right)$ and will continue to have equilibrium beliefs that $v_{i}<f_{j}\left(v_{j}\right)$. If, in addition, $v_{i}<\max _{v>v_{i}} f_{j}(v)$, then buyer $i$ would not know that $v_{i} \geq f_{j}\left(v_{j}\right)$ and will continue to have equilibrium beliefs; however, if $v_{i} \geq \max _{v>v_{i}} f_{j}(v)$, then buyer $i$ knows that $v_{i} \geq f_{j}\left(v_{j}\right)$ and would believe that $v_{j}=1$ (we arbitrarily choose this belief from $v_{j} \geq v_{i}$ ) and bid his value.

Thus, if $\phi$ or $r$ is sent instead of $v$, the winning bid will be less than $v_{l}$. If $v$ or $\phi$ is sent instead of $r$, the winning bid will be $f_{i}\left(v_{i}\right)$ where $i$ is the high-value buyer. Therefore, such beliefs and strategies gives no individual player incentive to deviate.

An example of such an equilibria with a uniform distribution is shown in Figure 4 . In this equilibrium, the correct values are sent if $v_{l}>.5 v_{h}$. Otherwise, the high-low signal is sent. The winning bid is each to $v_{l}$ in the first region and $v_{h} / 2$ in the other region. 


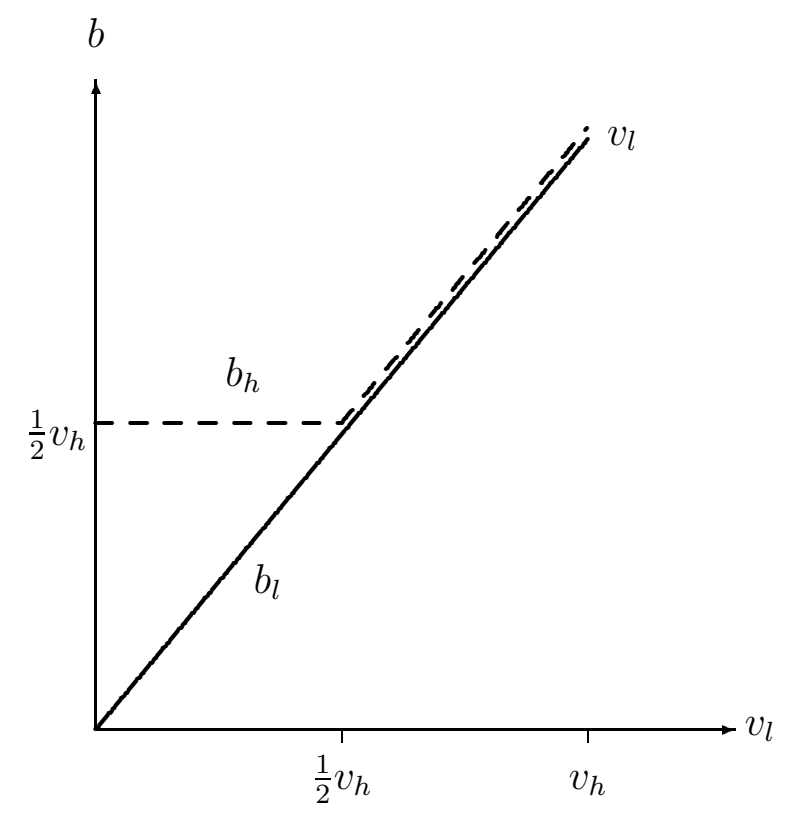

Figure 1: Equilibrium bid functions.

Proposition 4.2 Any equilibrium of the game $\Gamma_{p t}$ must satisfy the following. There must exist two functions $f_{1}$ and $f_{2}$ such that $f_{1}(v) \in a(v)$ and $f_{2}(v) \in a(v)$ for all $v \in[0,1]$, where $a(v)$ is defined in Lemma 4.9, and:

- Given his information $v=\left(v_{1}, v_{2}\right)$ the seller sends $v$ if $v_{1} \geq v_{2} \geq f_{1}\left(v_{1}\right)$ or $v_{2} \geq v_{1} \geq f_{2}\left(v_{2}\right)$, otherwise he sends $r$ or $\phi$ (which of these is sent may depend upon $v$ ).

- The auction is won always by the high valuation buyer $i$ who pays $v_{j}$ when $v$ is announced and $f_{i}\left(v_{i}\right)$ otherwise.

Proof: An equilibrium that did not satisfy the above conditions would violate the conditions set forth in Lemmatta 4.6, 4.7, 4.8, and 4.9.

Remark: The following is an equilibrium in which $\phi$ is sent as part of the seller's signaling strategy.

- Seller's strategy: when $v_{h} \geq .8$ and $v_{l} \leq .2$, the seller sends $\phi$. When $v_{h}<.8$ and $v_{l} \leq(1 / 2) v_{h}$, the seller sends $r$. Otherwise, he sends $v$. (See Figure 4.)

- Buyers' strategy: when $v_{h}<.8$, a buyer receiving $H$ bids $v_{i} / 2$. When $v_{l}<.4$, a buyer receiving $L$ bids $v_{i}$. A buyer with $v_{i} \leq .2$ receiving $\phi$ bids $v_{i}$ with probability $1 / 3$ and 0 with probability $2 / 3$. A buyer with $v_{i}>.8$ receiving $\phi$ bids .2. 


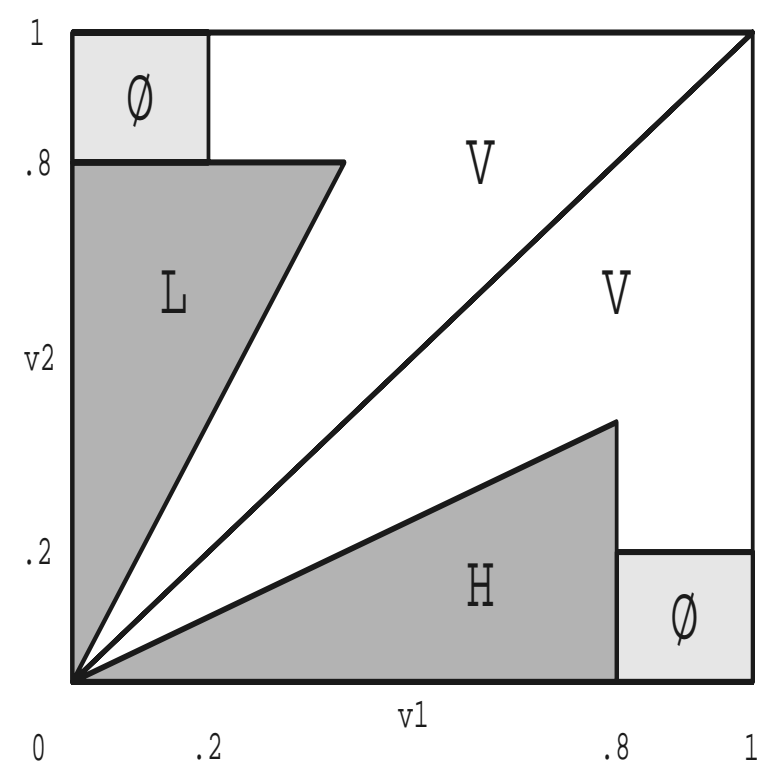

Figure 2: Example of when $\phi$ is sent in equilibrium.

- Off equilibrium: a buyer receiving $\phi$ when $.2<v_{i}<.8$ still believes that $v_{-i} \leq .2$ and bids $\left(v_{i}-.4\right) / 2$ for $v_{i}>.4$ and $\epsilon$ for $.2<v_{i} \leq .4$. A buyer receiving $H$ when $v_{h}>.8$ bids .2 (believes $v_{l}<.2$ ). A buyer receiving $L$ when $v_{l}>.4$ believes that $v_{h}$ is uniform between $v_{l}$ and 1 and bids accordingly (less than $v_{l}$ ).

Proof: We see that this is an equilibrium with the following. There is no $v$ for which the seller would like to switch his signal. Let us check all the possibilities.

- Sending $v$ instead of $r$ : this could only lower the winning bid from $v_{h} / 2$ to $v_{l}$.

- Sending $\phi$ instead of $r$ : if $v_{h}<.2$, this would change the winning bid to $(1 / 3) v_{h}+(2 / 9) v_{l}$ which is lower than $v_{h} / 2$ since $v_{l} \leq v_{h} / 2$; if $v_{h}>.2$, the winning bid is either $v_{l}$ or $\left(v_{h}-.4\right) / 2$, however both of these are less than $v_{h} / 2$, the winning bid if $r$ is sent.

- Sending $r$ instead of $\phi$ : this will keep the winning bid at .2.

- Sending $v$ instead of $\phi$ : this will only lower the winning bid from .2 to $v_{l}$.

- Sending $r$ instead of $v$ : this will lower the winning bid to either $v_{h} / 2$ if $v_{h}<.8$ or to .2 otherwise.

- Sending $\phi$ instead of $v$ : if $v_{l}<v_{h}<.2$, sending $\phi$ will generate expected revenue of $(1 / 4) v_{h}+(3 / 16) v_{l}$ which is less than $v_{l}$ since $v_{h}<2 v_{l}$; if $v_{h}>.2$, the winning bid is either $v_{l}$ or $\left(v_{h}-.4\right) / 2$ and can only lead to an increase if $\left(v_{h}-.4\right) / 2 \geq v_{l}$, however this cannot happen since $v_{h} / 2<v_{l}$. 
There is no signal sent for which the buyers would like to switch their strategies.

- When $v$ is sent: behavior is as shown in Lemma 4.1.

- When $r$ is sent: behavior is as shown in Lemma 4.4.

- When $\phi$ is sent: in equilibrium, $H$ knows he is high and knows that $L$ is bidding $v_{i}$ with probability $2 / 3$ and 0 with probability $1 / 3$. If $H$ bids .2 in equilibrium, he wins with certainty. If $H$ bids $x<.2$, he has expected payoff of $((1 / 3)(x / .2)+(2 / 3))(v-x)$. This is increasing in $x$ when $v \geq 2 x+.4$. Thus, when $v \geq .8$, buyer $H$ bids .2. Out of equilibrium, only a buyer with value $.2<v_{i}<.8$ knows he is out of equilibrium and believes that the other buyer has value between 0 and .2. His expected payoff is as previously mentioned and is maximized by bidding $\max \{(v-.4) / 2, \epsilon\}$.

Remark: The revenue for an equilibrium described in Proposition 4.1 with regions determined by $f_{1}(v)$ and $f_{2}(v)$ is higher than the revenue for an equilibrium with regions determined by $f_{1}^{\prime}\left(v_{h}\right)$ and $f_{2}^{\prime}(v)$ if $f_{i}$ is pointwise greater than $f_{i}^{\prime}$ for $i=1,2$.

Proposition 4.3 In the uniform case, the highest expected revenue of the seller is $5 / 12$.

Proof: Using the previous remark, the equilibrium with $f_{1}(v)=f_{2}(v)=(1 / 2) v$ is the equilibrium that yields the highest expected revenue for the seller. ${ }^{11}$ Given $v_{h}=x$, with probability $1 / 2$, the signal $r$ is sent and the selling price is $x / 2$ and with probability $1 / 2$, the signal $v$ is sent and the expected payoff (the conditional density of $v_{l}$ is $\left.2 / x\right)$ is $\int_{\frac{x}{2}}^{x} \frac{2}{x} y d y=3 / 4 \cdot x$. Hence, given $v_{l}=x$ the expected payoff is $1 / 2 \cdot x / 2+1 / 2 \cdot 3 / 4 \cdot x=5 / 8 \cdot x$ and the total revenue is $\int_{0}^{1}(5 / 8) x \cdot 2 x \cdot d x=5 / 12$.

\section{Full Commitment}

We will now see that the seller can generate strictly higher profits under full commitment power than under the cases of partial or no commitment power studied thus far. Using the same strategy sets and interpretation function as in the last section, we can see that a seller with full commitment power is able to

\footnotetext{
${ }^{11}$ Note that the expected revenue here is also the revenue of the highest revenue mechanism in the absense of seller information for the uniform case. One such a mechanism is a second-price auction with a reserve price of $1 / 2$. This equivalence of revenue is by numerical coincidence since for other distributions the revenue may be higher or lower than the optimal mechanism without seller information.
} 
announce more than just the rankings or values. For instance, within the same alphabet and interpretation function the seller can now send the maximum of the two values by sending $\left(0, \max \left\{v_{1}, v_{2}\right\}\right)$ (this is interpreted as a point in $V$, i.e. $v_{1}=0, v_{2}=\max \left\{v_{1}, v_{2}\right\}$ which is (almost surely) not a truthful message).

We will now see that this strategy of publicly announcing the value of the high-value buyer will result in higher profits for the seller. ${ }^{12}$ In such a case, the low-value buyer knows both values, while the high-value buyer knows only that he has the highest value.

We retain our Assumption 4.1 that a buyer does not bid above his value.

Proposition 5.1 There is a unique equilibrium in the game in which the seller commits himself to send (publicly) the signal $\max \left\{v_{1}, v_{2}\right\}$. In this equilibrium, high-value buyer uses a mixed strategy with continuous bid distribution with support $[\underline{b}, \bar{b}]$ where $\underline{b}<\bar{b}<v_{h}$. The low-value buyer uses a pure, increasing bidding strategy in the range $[\underline{b}, \bar{b}]$.

The proof is given in the Appendix.

Proposition 5.2 When $V_{1}$ and $V_{2}$ are uniformly (and independently) distributed on $[0,1]$, and the seller publicly announces the value of the high-value buyer, the following is an equilibrium: the high-value buyer uses the mixed-strategy with

$$
F(b)=\frac{e^{2}}{2} \cdot \frac{v_{h}}{2 b-v_{h}} \cdot e^{-\frac{v_{h}}{2 b-v_{h}}}
$$

on the support $\left[(1 / 2) v_{h},(3 / 4) v_{h}\right]$ and the low-value buyer uses the (pure) bid function $b_{l}\left(v_{l} ; v_{h}\right)=v_{h}-v_{h}^{2} /\left(4 v_{l}\right)$ when $v_{l} \geq(1 / 2) v_{h}$ and bids $v_{l}$ if $v_{l}<(1 / 2) v_{h}$.

The proof is given in the Appendix.

The analytical computation of the profits in the equilibrium of the uniform distribution case, is done in the Appendix and summarized in the following proposition (we denote $\left.\varphi(z)=\int_{z}^{\infty} \frac{e^{-t}}{t} d t\right)$ :

Proposition 5.3 When $V_{1}$ and $V_{2}$ are uniformly (and independently) distributed on $[0,1]$, and the seller publicly announces the value of the high-value buyer, the resulting equilibrium will yields:

(i) Expected seller revenue of $1 / 2-e \cdot \varphi(1) / 12 \approx .450304$.

(ii) Expected low-value buyer profits of $e \cdot \varphi(1) / 24 \approx .02485$.

(iii) Expected high-value buyer profits of $1 / 6$.

(iv) Welfare loss of $e \cdot \varphi(1) / 24 \approx .02485$ (total expected surplus minus total possible surplus).

\footnotetext{
${ }^{12}$ We do not yet know what strategy yields the highest profits.
} 
Remark While the equilibrium established here is for full commitment power, it can be easily made an equilibrium of a full truthful commitment model by slightly modifying the interpretation function as follows (see Proposition 2.4):

As under the usual interpretation function $I((h, l))=\left\{\{v\} \mid v \in V\right.$ and $v_{1} \geq$ $\left.v_{2}\right\}$, and $I((l, h))=\left\{\{v\} \mid v \in V\right.$ and $\left.v_{1}<v_{2}\right\}$. However, $I\left(\left(v_{1}, v_{2}\right)\right)=\left\{\left(v_{1}, v_{2}\right)\right\}$ only when $v_{1}>0$. Otherwise, $I\left(\left(0, v_{m}\right)\right)=\left\{\{v\} \mid v \in V\right.$ and either $v_{1} \leq v_{m}$ and $v_{2}=$ $v_{m}$ or $v_{2} \leq v_{m}$ and $\left.v_{1}=v_{m}\right\}$.

We conjecture that our results can also show that under full truthful commitment power the seller's revenue can be higher than partial truthful commitment power. We believe that the addition of two other signals will not lead to additional equilibrium allocations under partial commitment power. These two other signals are $\max \left\{v_{1}, v_{2}\right\}$ and $\min \left\{v_{1}, v_{2}\right\}$. That is the seller is able to announce the highest value or the lowest value with the buyers aware whether the announced value is the highest. This would imply that the above mentioned seller strategy would also be a valid seller strategy with full truthful commitment power and a signal set that has the same equilibrium allocations as in the partial truthful commitment section. Thus, we provide a case where moving from partial truthful commitment power to full truthful commitment power increases seller revenue.

We present the logic for the above conjecture in the following. In any partial truthful commitment equilibrium, announcing $\max \left\{v_{1}, v_{2}\right\}$ is equivalent to sending the rankings, while announcing $\min \left\{v_{1}, v_{2}\right\}$ is equivalent to sending the values. The reason for this is that, in equilibrium, the winning bid will be greater than $\min \left\{v_{1}, v_{2}\right\}$ otherwise the seller would have incentive to send the values. When this is sent, the equilibrium would have the high-value buyer win the auction at the lowest value. For similar logic as presented in the partial truthful commitment section, the high-value buyer must win at the highest of the possible low-values he may face. Announcing $\max \left\{v_{1}, v_{2}\right\}$ will inform the buyers of the rankings and the $L$ buyer of the $H$ buyer's value. This will not help the $L$, buyer, since the $H$ buyer will still win. The only question is whether there can be two regions (as with $r$ and $\phi$ ): one where the rankings is sent and one where max is sent. Since the seller can send $(h, l)$ instead of $\max \left\{v_{1}, v_{2}\right\}=v_{1}$ and vis-versa, the high-value buyer's bid must be the same in either region. Thus, the set of equilibrium outcomes is equivalent to before.

\section{Discussion}

\subsection{Comparison among environments}

The expected profits to the seller, low-value buyer and high-value buyer are displayed in Figure 3 for the case of uniform distribution. The results of LRWZ [1998] showed when the buyers are informed of their rankings $(b)$, the expected profit for the seller is higher than in the standard first-price auction $(a)$. The 


\begin{tabular}{|l|l|l|l|l|l|}
\hline & Model & Seller & $\begin{array}{l}\text { Low } \\
\text { Buyer }\end{array}$ & $\begin{array}{l}\text { High } \\
\text { Buyer }\end{array}$ & $\begin{array}{l}\text { Welfare } \\
\text { Loss }\end{array}$ \\
\hline a & Standard First-Price & 0.3333 & 0 & 0.3333 & 0 \\
b & Rankings Known & 0.3696 & 0.0437 & 0.227 & 0.0264 \\
c & No Commitment & 0.3333 & 0 & 0.3333 & 0 \\
d & Partial Commitment & 0.4167 & 0 & 0.25 & 0 \\
e & Full Commitment & 0.4503 & 0.02485 & 0.1667 & 0.02485 \\
\hline
\end{tabular}

Figure 3: Summary of Expected Profits in the uniform distribution case

auction is no longer efficient, and this inefficiency is measured by the 'Welfare Loss' in the last column of Figure 3 which is defined as the total expected surplus (buyers' plus the seller's) minus total possible surplus.

Our first results indicated that without commitment power $(c)$, the seller could not take advantage of the knowledge of the rankings and the equilibria would be equivalent to that of case $(a)$. Only with full commitment power can the seller obtain the expected revenue of model $(b)$.

Our next set of results are for the environment in which the seller knows the values. With partial commitment power $(d)$, the seller can generate more revenue than in the case in which he has no information $(a)$. This is even higher than the expected revenue when the buyers know the rankings (b). Surprisingly, the equilibrium of this model is efficient (no welfare loss).

While partial commitment power $(d)$ is useful to the seller, with full commitment $(e)$ the seller can generate even higher revenues using the strategy described in the previous section. However, in generating higher revenue, the induced equilibrium loses the efficiency property.

On the buyer side, we see that with full commitment $(e)$ the low-value buyer has additional information $v_{h}$. In equilibrium this decreases payoffs of both buyers compared to the situation $(b)$ in which both players just know the rankings. The low-value buyer would rather commit to not looking at $v_{h}$ but he cannot credibly do so (it cannot be part of a sequential equilibrium).

This type of situation occurs elsewhere in economics. For instance, in a Cournot duopoly, if the (Stackelberg) leader credibly transmits his output quantity, the follower would do better by committing to not looking at the information; however, without a commitment power, the follower would not be credible in saying he 'would not look at the leader's information'; it is not a subgame perfect equilibrium. 


\subsection{Relationship to Classic Results}

Our results stand in contrast to two landmark results of auction theory: revenue equivalence in private-value auctions (Myerson [1981] and Riley and Samuelson [1981]) and optimality of full revelation of the seller's information (Milgrom and Weber [1982a, 1982b]).

As we saw in our framework, two efficient private-value auctions need not have the same revenue. In the partial commitment model, the equilibrium is efficient yet expected revenue is higher than that in the standard first or second price auction $(5 / 12=0.4167$ compared to $1 / 3$, for the uniform case). As to the revelation of information by the seller, Milgrom and Weber have an implicit assumption that the seller has full commitment power, so any signal by him is credible. ${ }^{13}$ The appropriate comparison is therefore to our full commitment framework and there we find that in equilibrium when the seller reveals his information (the realized values) only to the low-value buyer, the expected revenue is higher than if the seller would commit to reveal publicly all his information (0.4503 compared to $1 / 3$, in the uniform case).

More surprisingly is the fact that except one assumption, our model can be viewed as a special case of the Milgrom-Weber general model. They assume:

- Each buyer $i$ has a private signal $X_{i}$ (which is $v_{i}$ in our case).

- The seller has a private signal $S$.

- There is symmetry of bidder utility functions, $u_{i}(s, x)=u\left(s, x_{i},\left\{x_{j}\right\}_{j \neq i}\right)$ (which is just $x_{i}$ in our case). Utility $u_{i}$ is symmetric in the other bidders' signals $\left\{x_{j}\right\}_{j \neq i}$ (in our case it is independent of these other signals). The buyers' utility is continuous and non-decreasing in its variables.

- $S_{1}, \ldots, S_{m}, X_{1}, \ldots, X_{n}$ are affiliated.

- The distribution density $f(s, x)$ of $(S, X)$ is symmetric in $X\left(=\left(X_{1}, \ldots, X_{n}\right)\right)$.

It is only this last assumption which is not satisfied in our model. For instance, when the seller's information is $H=$ "buyer 1 has the highest valuation," then clearly the density of $\left(H ; v_{1}, v_{2}\right)$ is not symmetric in $\left(v_{1}, v_{2}\right)$ as it is concentrated on $\left\{\left(v_{1}, v_{2}\right) \mid v_{1} \geq v_{2}\right\}$.

These observations about the relationship of our model to the Milgrom-Weber model indicate that their assumption of the symmetry of distribution is a very crucial one. A more general framework with its relaxation could lead to important results.

\footnotetext{
${ }^{13}$ In future work, we wish to investigate in which way will the relaxation of this assumption, to partial commitment or no commitment power, affect their results.
} 


\subsection{Relationship to the Crawford-Sobel Model}

In "Strategic Information Transmission," Crawford and Sobel [1982] present a model of two players: a sender and a receiver. The sender knows the state of nature $s$ (in $[0,1])$ and sends a message $m=m(s)$ to the receiver who chooses an action $y=y(m)$. The sender and receiver have utilities that differ via a parameter $b$; the sender's utility is $-(y-s-b)^{2}$, while the receiver's utility is $-(y-s)^{2}$.

They find that without commitment power, each equilibrium is defined by a partition $\pi$ of $[0,1]$ where the sender sends $\pi(s)$, the partition element containing $s$. The partition sent never fully reveals the state since all partitions contain multiple states $\{s\} \neq \pi(s)$. In such an equilibrium, both parties gain in expected payoffs (over the no information model) if there are at least two elements in $\pi$.

We can compare this to our model if the message space (alphabet) and interpretation function are such that for any state $s$ in $[0,1]$ there is a message $m$ such that $I(m)=\{s\}$. Using our definitions of the various commitment levels, we find that with partial truthful or full commitment power, there is full revelation in equilibrium, $m(s)=s$ and $y(m)=m$. In this full-revelation equilibrium, both parties have higher expected payoffs than in any of the Crawford-Sobel equilibria.

In other words, the sender in the Crawford-Sobel model reveals information partially and not fully (by sending an interval containing the state and not the state $s$ itself) only because of lack of commitment power and not for strategic reasons. In our model, even with full commitment power the sender (seller) reveals his information only partially, for strategic reasons.

Seidmann and Winter [1997] apply the Milgrom [1981] version of partial truthful commitment to the Crawford-Sobel model and arrive at similar results to what we present in this section. They also provide an example of a sender-receiver game that is similar to the Crawford-Sobel model that has, as with our model, partial disclosure of information with partial truthful commitment.

We provide the following proposition that confirms the above application of our notions of commitment power to the Crawford-Sobel model (the proof for partial truthful case is substantially shorter than Seidmann and Winter due to our use of a more specific environment).

Proposition 6.1 If for each state of nature there is at least one available message which is truthful only at that state (hence such a message fully reveals the state of nature), then under partial truthful, full truthful and full commitment power, full revelation is the only equilibrium outcome.

Proof: Assume that in equilibrium, at $s \in[0,1]$ the sender sends a message $m(s)$. Let $M_{s}=\{\tilde{s} \mid m(\tilde{s})=m(s)\}$ (note that $M_{\tilde{s}}=M_{s}$ for all $\tilde{s} \in M_{s}$ ). We claim that $M_{s}=\{s\}$. Let $y\left(M_{s}\right)=y(m(s))$ be the action of the receiver when he receives $m(s)$. First observe that since the sender can guarantee $-b^{2}$ at any 
$\tilde{s} \in M_{s}$ (by sending $\left.M(\tilde{s})=\{\tilde{s}\}\right)$, it must be that $-\left(y\left(M_{s}\right)-\tilde{s}-b\right)^{2} \geq-b^{2}$ for all $\tilde{s} \in M_{s}$ implying:

$$
y\left(M_{s}\right) \geq \tilde{s} \text { for all } \tilde{s} \in M_{s}
$$

On the other hand,

$$
y\left(M_{s}\right)=\arg \max _{y} E\left[-(y-\tilde{s})^{2} \mid \tilde{s} \in M_{s}\right]=E\left[\tilde{s} \mid \tilde{s} \in M_{s}\right]
$$

Therefore,

$$
E\left[\tilde{s} \mid \tilde{s} \in M_{s}\right] \geq \tilde{s} \text { for all } \tilde{s} \in M_{s}
$$

and hence $M_{s}=\{s\}$.

We now show that with full (or full truthful) commitment power, full revelation is also the only equilibrium outcome. If instead the sender chooses to send the same message for all states in set $M$, the receiver would choose $y(M)=E[s \mid s \in M]$. The expected utility of the sender (given that $s$ is in $M$ ) is

$$
E\left[-(y(M)-s-b)^{2} \mid s \in M\right]=E\left[-(s-E[s \mid s \in M])^{2} \mid s \in M\right]-b^{2}
$$

In equilibrium, this should be at least $-b^{2}$, since the seller can obtain a utility of $-b^{2}$ by sending $\{s\}$ for all $s$ in $M$. This implies that the set $M$ must be a singleton.

\section{Conclusion}

In this paper, we have begun the investigation of the strategic use of seller information in private-value auctions. This issue is not only intriguing theoretically, but is relevant to real situations such as procurement or privatization. Methodologically, our approach is in the spirit of the works of Kamien, Tauman, and Zamir (KTZ) [1990a and 1990b] on the value of information in a strategic conflict: 'injecting' information into a game, by public or private messages, changes the original game and induces a different game. The effect of the information is 'measured' by the 'outcome' or Nash equilibria of the induced game. The fundamental departure from the KTZ model is that while they consider an information holder who is an outside agent and not a player in the original game, we consider the seller as an information holder whose payoff is determined directly from the outcome of the game among the buyers.

Our principle conclusion is that this information is useful and that the usefulness is highly dependent upon the level of commitment power the seller possesses. We show this by examining the set of equilibria under different levels of commitment power with two possible different information conditions for the seller. Under both cases, the seller uses public signals only. In the first case, the seller knows which of the two buyers has the highest value (the rankings). In the second case, the seller knows the buyers' values completely. 
When only the rankings are known, we know from LRWZ that the seller can gain if the buyers become aware of their rankings. We find this gain only when the seller can commit to tell the truth or commit to a specific strategy (partial truthful, full truthful and full commitment). If the seller cannot commit, we find that under any alphabet of signals or any interpretation function of these signals, the seller cannot gain from his information and any equilibrium is equivalent to the no information equilibrium.

If, in addition, the values are known to the seller and he is able to announce either the rankings, values, the higher value, or the lower value, then the seller can gain from having partial truthful commitment power even more than with full commitment power when he knows only the rankings. Furthermore, when he has full commitment power, he can gain even more.

In future work, we plan to look further at the no commitment case. Preliminary results seem to indicate that when the seller has full information he cannot gain from his information without some commitment power, however, under a different information partition he can gain from his information even with no commitment power. We also plan to study the partial truthful commitment case under a larger set of signals to see if the seller can do strictly better. As mentioned in the introduction, we also study the question of seller information in the framework of mechanism design (Kaplan and Zamir, 2000).

\section{References}

Crawford, V., and J. Sobel (1982): "Strategic Information Transmission," Econometrica, 50(6), 1431-1451.

Farrell, J., and R. Gibbons (1989): "Cheap Talk with Two Audiences," American Economic Review, 95(5), 1214-1223.

Kaplan, T., and S. Zamir (2000): "Optimal Auction Design with an Informed Seller," Working paper.

Landsberger, M., J. Rubinstein, E. Wolfstetter, and S. Zamir (1998): "First-Price Auctions When the Ranking of Valuations is Common Knowledge," Hebrew University of Jerusalem Discussion Paper.

Milgrom, P. R. (1981): "Good News and Bad News: Representation Theorems and Applications," Bell Journal of Economics, 21, 380-391.

Milgrom, P. R., and J. Roberts (1986): "Relying on the Information of Interested Parties," Rand Journal of Economics, 17(1), 18-32.

Milgrom, P. R., and R. J. Weber (1982a): "A Theory of Auctions and Competitive Bidding," Econometrica, 50(5), 1089-1122. 
- (1982b): "The Value of Information in a Sealed-Bid Auction," Journal of Mathematical Economics, 10(1), 105-114.

Morton Kamien, Yair Tauman, S. Z. (1990a): "Information Transmission," in Game Theory and Applications, ed. by Y. T. Tatsuro Ichiishi, Abraham Neyman, pp. 273-281. Academic Press.

(1990b): "On the Value of Information in a Strategic Conflict," Games and Economic Behavior, 2(2), 129-153.

Myerson, R. B. (1981): "Optimal Auction Design," Mathematics of Operations Research, 1(1), 58-73.

Riley, J., and W. Samuelson (1981): "Optimal Auctions," American Economic Review, 71(3), 381-392.

Seidmann, D., and E. Winter (1997): "Strategic Information Transmission with Verifiable Messages," Econometrica, 65(1), 163-170.

\section{A Appendix}

\section{A.1 Proof of Lemma 4.1}

Consider first the case $v_{l}<v_{h}$ and let $\underline{l}=\inf \left\{b \mid P\left(b_{l}<b\right)>0\right\}$. Thus, $\underline{l}$ is the lower bound of the support of $L$ 's strategy. First observe that $P\left(b_{h}<\underline{l}\right)=0$ since $b_{h}<\underline{l}$ yields zero profit while $H$ can guarantee positive profit by any bid $b$ satisfying $v_{l}<b<v_{h}$. For the same reason,

$$
P\left(b_{l}=\underline{l}\right)=0 \text { implies } P\left(b_{h} \leq \underline{l}\right)=0
$$

We claim first that the expected payoff of $L$ in equilibrium is zero. In fact, this is true if $\underline{l}=v_{l}$, since then (by Assumption 4.1 and the definition of $\underline{l}$ ) $P\left(b_{l}=v_{l}\right)=1$ and $L$ never has a positive payoff. If $\underline{l}<v_{l}$, then $P\left(b_{h}=\underline{l}\right)=0$ since otherwise (by (10)) $P\left(b_{l}=\underline{l}\right)>0$ which is impossible (both buyers cannot play an atom at the same point $\underline{l})$.

Hence, $P\left(b_{h}>\underline{l}\right)=1$ and consequently $P\left(L\right.$ wins $\left.\mid b_{l}=\underline{l}\right)=0$ implying that, again, his expected payoff is zero (since $\underline{l}$ is in the support of his strategy).

Next we claim that $\underline{l}=v_{l}$ is impossible in equilibrium since buyer $H$ has no best reply. (Clearly $P\left(b_{h}<v_{l}\right)=0$ and to any strategy with $P\left(b_{h}>v_{l}\right)>0$ there is a profitable deviation, but to $b_{h}=v_{l}$ there is also a profitable deviation.)

We conclude that $\underline{l}<v_{l}$, and $P\left(b_{h} \geq v_{l}\right)=1$ (otherwise $L$ would have a deviation with positive expected payoff). Since again $P\left(b_{h}>v_{l}\right)>0$ is impossible, we must have $b_{h}=v_{l}$ and finally $P\left(b_{l}<v_{l}\right)=1$ (otherwise $H$ would have a profitable deviation). 
For $v_{l}=v_{h}=v$, using standard arguments, it is readily verified that the only pure-strategy equilibrium is $b_{1}=b_{2}=v$. Since bids are bounded below by zero, there is not a possibility of a mixed-strategy equilibrium (see Kaplan \& Wettstein [2000]).

\section{A.2 Proof of Proposition 5.1}

Denote $\Gamma_{v_{h}}$ the game in which the seller commits himself to send (publicly) the signal $v_{h}=\max \left\{v_{1}, v_{2}\right\}$.

Lemma A.1 In game $\Gamma_{v_{h}}$, high-value buyer cannot use a pure strategy in equilibrium.

Proof: Assume to the contrary that there is some $v_{h}>0$ at which the high-value buyer bids a pure strategy, say $b_{h}$. In equilibrium, $b_{h}<v_{h}$, since the high-value buyer makes zero profits with $b_{h}=v_{h}$ and can make strictly positive profits with any $b_{h}$ strictly between 0 and $v_{h}$ (he would win $v_{h}-b_{h}$ whenever $v_{l}<b_{h}$ ). The low-value buyer, knowing $v_{h}$, would therefore know $b_{h}$. To maximize his profit, whenever $v_{l}>b_{h}$, he would choose the lowest bid strictly greater than $b_{h}$. Such a bid does not exist. Therefore, the high-value buyer cannot use this pure strategy. ${ }^{14}$

Lemma A.2 In the equilibrium of game $\Gamma_{v_{h}}$, the mixed-strategy of the high-value buyer cannot have any atoms nor gaps in its support.

Proof: First, let us show that there are no atoms. If there are, consider the highest of such atoms. Using the arguments in the proof of Lemma A.1, this atom must be below $v_{h}$. Given this, there must be some positive measure of low-value buyers that would wish to bid slightly above this atom. The rest of the argument is again as before in the proof of Lemma A.1.

Second, assume that $a$ and $b$ are in the support of $H$ 's strategy, while the interval $(a, b)$ is not. Then no $L$ buyer bids in $(a, b)$, since bidding $a$ is strictly better. Consequently for $H$ also bidding $a$ is strictly better than $b$ in contradiction to the fact that they are both in the support of the equilibrium strategy.

Lemma A.3 In the equilibrium of game $\Gamma_{v_{h}}$, the low-value buyer must bid a continuous strictly increasing function of his value on the support of the highvalue buyer.

\footnotetext{
${ }^{14}$ One can also see that this is not an artifact of a point existence problem. All the low-value buyers with values strictly greater than $b_{h}$ would want to choose the same point 'slightly' above $b_{h}$. The high-value buyer would then have incentive to move to a bid higher than such a point.
} 
Proof: Given the value $v_{h}$ of $H$, let $[\underline{b}, \bar{b}]$ be the support of the mixed strategy in equilibrium. Note that $0 \leq \underline{b}<\bar{b}<v_{h}$ (since $H$ has positive expected payoff in equilibrium) and by Lemma A.2, buyer H's strategy has no atoms nor gaps in $[\underline{b}, \bar{b}]$. Consequently, the probability $P(b)=P\left(b>b_{h}\right)$ that $L$ wins with a bid $b$ is strictly increasing in $b$ in the interval $\underline{b} \leq b \leq \bar{b}$.

Let $b_{l}\left(v_{l}\right)$ be the (possibly random) bid of the $L$ buyer when his value is $v_{l} \leq v_{h}$. Note that an $L$ buyer with value $v_{l}>\underline{b}$ expects a positive payoff in equilibrium (since a positive payoff is guaranteed by bidding, for example, $\left(v_{l}+\underline{b}\right) / 2$ ). Hence, the support of $b_{l}\left(v_{l}\right)$ is a subset of $[\underline{b}, \bar{b}]$ (any lower bid yields zero payoff and any higher bid is strictly dominated by $\bar{b}$ ). Let $\beta=b_{l}\left(V_{l}\right)$ be the random bid of the (random) $L$ buyer in equilibrium.

We first claim that there are no gaps in the cumulative distribution of $\beta$ in $[\underline{b}, \bar{b}]$. Assume there is such a gap $(x, y)$ where $\underline{b} \leq x<y \leq \bar{b}$. Then, the probability $P\left(b_{l} \leq z\right)$ is the same for all $z$ in $(x, y)$. Clearly, $H$ would strictly prefer the lowest of such points implying $(x, y]$ is not in the support of his strategy in contradiction to Lemma A.2. Similarly there cannot be an atom in the distribution of $\beta$ in $(\underline{b}, \bar{b}]$ since if there is an atom, say at $\alpha \in(\underline{b}, \bar{b}]$, then for bidder $H$, for sufficiently small $\epsilon>0$, any bid in the interval $(\alpha-\epsilon, \alpha)$ is strictly dominated by the bid $\alpha+\epsilon / 2$, in contradiction to the fact that the mixed strategy of $H$ has no gaps. Next we claim that for all $v_{l} \in\left[\underline{b}, v_{h}\right]$ the bidding strategy $b_{l}\left(v_{l}\right)$ is pure. Assume to the contrary that for a certain $v_{l}^{\prime} \in\left[\underline{b}, v_{h}\right]$, two different bids $b_{1}$ and $b_{2}$, where $\underline{b}<b_{1}<b_{2}<, \bar{b}$, are both in the support of $b_{l}\left(v_{l}^{\prime}\right)$. Then this $L$ buyer must be indifferent between these two bids and weakly prefer them to any bid in between them. Thus,

$$
P\left(b_{1}\right)\left(v_{l}^{\prime}-b_{1}\right)=P\left(b_{2}\right)\left(v_{l}^{\prime}-b_{2}\right) \geq P(x)\left(v_{l}^{\prime}-x\right) \text { for all } x \text { in }\left(b_{1}, b_{2}\right)
$$

where $P\left(b_{i}\right)$ is the probability of winning the auction with bid $b_{i}$.

For all $v_{l}^{\prime \prime}>v_{l}^{\prime}$, the bid $b_{2}$ is strictly preferred to any bid $x$ in $\left[b_{1}, b_{2}\right)$, since

$$
\begin{aligned}
P\left(b_{2}\right)\left(v_{l}^{\prime \prime}-b_{2}\right)=P\left(b_{2}\right)\left(v_{l}^{\prime}-b_{2}\right)+P\left(b_{2}\right)\left(v_{l}^{\prime \prime}-v_{l}^{\prime}\right) & \geq \\
P(x)\left(v_{l}^{\prime \prime}-x\right)+\left(P\left(b_{2}\right)-P(x)\right)\left(v_{l}^{\prime \prime}-v_{l}^{\prime}\right) & >P(x)\left(v_{l}^{\prime \prime}-x\right) \quad \forall x<b_{2}
\end{aligned}
$$

(from $P(x)$ being strictly increasing).

Likewise, for all $v_{l}^{\prime \prime}<v_{l}^{\prime}$, the bid $b_{1}$ is strictly preferred to all bids $x$ in $\left(b_{1}, b_{2}\right]$. Thus, any $L$ buyer with value $v_{l} \neq v_{l}^{\prime}$ in the interval $\left[\underline{b}, v_{h}\right]$, bids with probability zero in $\left(b_{1}, b_{2}\right)$ and thus $P\left\{\beta \in\left(b_{1}, b_{2}\right)\right\}=0$ in contradiction to the fact that the distribution of $\beta$ has no gap in $[\underline{b}, \bar{b}]$.

We proceed now to show that the (pure) bidding strategy of $L, b_{i}(v)$ is strictly increasing. Let $F$ be the cumulative distribution of the $H$ buyer's bidding strategy, and let $v_{1}>v_{2}$ be two values of $v_{l}$. First we claim that $b_{l}\left(v_{1}\right) \neq b_{l}\left(v_{2}\right)$ since if they were both equal, say to $b$, then we would have $b_{l}(v)=b$ for all $v \in\left(v_{1}, v_{2}\right)$ (since for all $b, F(b)\left(v_{2}-b\right) \leq F(b)(v-b) \leq F(b)\left(v_{1}-b\right)$, and hence $F(b)(v-b)$ 
has the same maximum point as the other two functions), in contradiction to the fact that the distribution of $\beta$ has no atoms. By the equilibrium conditions:

$$
F\left(b_{l}\left(v_{1}\right)\right) \cdot\left(v_{1}-b_{l}\left(v_{1}\right)\right) \geq F\left(b_{l}\left(v_{2}\right)\right) \cdot\left(v_{1}-b_{l}\left(v_{2}\right)\right)
$$

and

$$
F\left(b_{l}\left(v_{2}\right)\right) \cdot\left(v_{2}-b_{l}\left(v_{2}\right)\right) \geq F\left(b_{l}\left(v_{1}\right)\right) \cdot\left(v_{2}-b_{l}\left(v_{1}\right)\right)
$$

This implies that $F\left(b_{l}\left(v_{1}\right)\right)\left(v_{1}-v_{2}\right) \geq F\left(b_{l}\left(v_{2}\right)\right)\left(v_{1}-v_{2}\right)$ and consequently $F\left(b_{l}\left(v_{1}\right)\right) \geq F\left(b_{l}\left(v_{2}\right)\right)$ (since $v_{1}>v_{2}$ ) and therefore $b_{l}\left(v_{1}\right) \geq b_{l}\left(v_{2}\right)$. Thus, $b_{l}$ is weakly increasing. However, $b_{l}$ must be strictly increasing on $(\underline{b}, \bar{b}]$ as well since if $b_{l}$ were not, there would be an atom in $\beta$. Finally, since $b_{l}$ is strictly increasing, it must also be continuous since otherwise the distribution of $\beta$ would have a gap.

\section{A.3 Proof of Proposition 5.2}

From Lemmatta A.1, A.2, and A.3, we know that $H$ uses a mixed strategy with no atoms nor gaps and $L$ uses a pure strategy according to an increasing, continuous function $b_{l}$. Again let $[\underline{b}, \bar{b}]$ be the support of the mixed strategy of buyer $H$ with value $v_{h}$.

Given $v_{h}$, we first show that $\underline{b} \leq v_{h} / 2$. Denote by $G(b)$ the (normalized) cumulative distribution of $L$ 's bid in equilibrium that is, (note that $\operatorname{Pr}\left\{v_{l}<v_{h}\right\}$ is the probability that a buyer's value, independent of whether he is high or low, is less than $v_{h}$ ),

$$
G(b)=\operatorname{Pr}\left\{b_{l}\left(v_{l}\right)<b \mid v_{l}<v_{h}\right\} \cdot \operatorname{Pr}\left\{v_{l}<v_{h}\right\}
$$

A buyer never bids higher than his value, which implies that

$$
G(b) \geq \operatorname{Pr}\left\{v_{l}<b \mid v_{l}<v_{h}\right\} \cdot \operatorname{Pr}\left\{v_{l}<v_{h}\right\}=b
$$

In particular, $G(\underline{b}) \geq \underline{b}$. In addition, we claim that $G(\underline{b}) \leq \underline{b}$ (and hence $G(\underline{b})=\underline{b}$ ). In fact, since $L$ knows $v_{h}$ he also knows $[\underline{b}, \bar{b}]$. If $v_{l}>\underline{b}$, he will never bid less than $\underline{b}$ (by which he would lose with certainty); bidding $\left(\underline{b}+v_{l}\right) / 2$ is a profitable deviation, thus $G(\underline{b}) \leq P\left(v_{l} \leq \underline{b}\right)=\underline{b}$. Now, since $\underline{b}$ is in the support of buyer $H$ 's strategy, he prefers it to any bid $b$ outside of his support. Thus, for all $b \leq \underline{b}$ we have:

$$
G(b)\left(v_{h}-b\right) \leq G(\underline{b})\left(v_{h}-\underline{b}\right)
$$

Since $G(\underline{b}) \leq \underline{b}$, we also have:

$$
G(b)\left(v_{h}-b\right) \leq \underline{b}\left(v_{h}-\underline{b}\right)
$$

and hence

$$
b\left(v_{h}-b\right) \leq G(b)\left(v_{h}-b\right) \leq \underline{b}\left(v_{h}-\underline{b}\right) \text { for all } b \leq \underline{b}
$$


This leads to a contradiction if $\underline{b}>v_{h} / 2$ since $b\left(v_{h}-b\right)$ attains a maximum at $b=v_{h} / 2$.

Buyer $H$ must be indifferent among all the bids in the support of his strategy; all yield the same expected profit, say $c$. Therefore, $\left(b_{l}^{-1}(b) / v_{h}\right) \cdot\left(v_{h}-b\right)=c$, which we rewrite as

$$
b_{l}^{-1}(b)=\frac{v_{h} \cdot c}{v_{h}-b}
$$

We will now determine buyer $L$ 's bid function on $[\underline{b}, \bar{b}]$. Since $G(\underline{b})=\underline{b}$, we have $b_{l}^{-1}(\underline{b})=\underline{b}$. We now show that $b_{l}^{-1}(\bar{b})=v_{h}$. If $b_{l}\left(v_{h}\right)>\bar{b}$, then buyer $L$ with value $v_{l}=v_{h}$ would have a profitable deviation to $b_{l}\left(v_{h}\right)-\epsilon$ (with which he still wins the auction with certainty). If $b_{l}\left(v_{h}\right)<\bar{b}$, then (since $b_{l}$ is monotone) $\bar{b}-\epsilon$ would also be a certain win for $H$ and hence $\bar{b}$ cannot be in the support of $H$ 's strategy in equilibrium. Thus, $b_{l}\left(v_{h}\right)=\bar{b}$.

Assuming that $b_{l}$ is differentiable at $\underline{b}$, we have $b_{l}^{-1^{\prime}}(\underline{b})=v_{h} \cdot c /\left(v_{h}-\underline{b}\right)^{2}=$ $\underline{b} /\left(v_{h}-\underline{b}\right)$. Since $L$ never bids above his value, $b_{l}^{-1^{\prime}}(\underline{b}) \geq 1$ and therefore, $\underline{b} \geq v_{h} / 2$. Since we also have $\underline{b} \leq v_{h} / 2$, we conclude that $\underline{b}=v_{h} / 2$. From this and $b_{l}^{-1}(\underline{b})=\underline{b}$, we have that $c=v_{h} / 4$, and substituting this into the condition $b_{l}^{-1}(\bar{b})=v_{h}$, yields $v_{h}=v_{h} \cdot\left(v_{h} / 4\right) /\left(v_{h}-\bar{b}\right)$. This determines the upper end of the support as $\bar{b}=(3 / 4) v_{h}$.

We conclude that we know that the support of buyer $H$ strategy is $\left[(1 / 2) v_{h},(3 / 4) v_{h}\right]$, and the bidding strategy of buyer $L$ for $v_{l} \geq v_{h} / 2$ is $b_{l}^{-1}(b)=\left(v_{h}^{2} / 4\right)\left(v_{h}-b\right)$, which is

$$
b_{l}=b_{l}\left(v_{l}, v_{h}\right)=v_{h}-\frac{v_{h}^{2}}{4 v_{l}}
$$

We can now solve for the cumulative density $F$ of buyer $H$, by looking at the equilibrium condition for buyer $L$ which is: $F(b)\left(v_{l}-b\right)$ is maximized at $b=b_{l}\left(v_{l}\right)$. This yields the following differential equation.

$$
\frac{F(b)}{F^{\prime}(b)}=b_{l}^{-1}(b)-b
$$

Substituting for $b_{l}^{-1}$, we get

$$
\frac{F(b)}{F^{\prime}(b)}=\frac{v_{h}^{2}}{4\left(v_{h}-b\right)}-b
$$

The solution to this equation, satisfying the boundary conditions $F\left(v_{h} / 2\right)=0$ and $F\left(3 v_{h} / 4\right)=1$, is

$$
F(b)=\frac{e^{2}}{2} \cdot \frac{v_{h}}{2 b-v_{h}} \cdot e^{-\frac{v_{h}}{2 b-v_{h}}}
$$




\section{A.4 Proof of Proposition 5.3}

In this section we calculate the expected revenues of the seller and the buyers in equilibrium when the seller has full commitment power and values are drawn uniformly.

Recall that in the equilibrium, the seller publicly announces the high valuation $v_{h}$. The high value buyer makes a random bid $b$ with support on $\left[1 / 2 v_{h}, 3 / 4 v_{h}\right]$ and cumulative distribution $F$ given by:

$$
F(b)=\frac{e^{2}}{2} \cdot \frac{v_{h}}{2 b-v_{h}} \cdot e^{-\frac{v_{h}}{2 b-v_{h}}}
$$

Hence, the density function is:

$$
f(b)=2 e^{2} \cdot \frac{v_{h}\left(v_{h}-b\right)}{\left(2 b-v_{h}\right)^{3}} \cdot e^{-\frac{v_{h}}{2 b-v_{h}}}
$$

The low-value buyer uses a pure bidding strategy:

$$
b_{l}= \begin{cases}v_{l} & \text { if } v_{l} \leq v_{h} / 2 \\ v_{h}-v_{h}^{2} / 4 v_{l} & \text { if } v_{h} / 2 \leq v_{l} \leq v_{h}\end{cases}
$$

We start by computing the conditional expected selling price given that the high value is $v_{h}$.

(i) If $0 \leq v_{l} \leq v_{h} / 2$, the buyer $H$ wins with certainty and the expected selling price is therefore the mean of his bid, which is

$$
\int_{\frac{v_{h}}{2}}^{\frac{3 v_{h}}{4}} b \cdot f(b) d b=\int_{\frac{v_{h}}{2}}^{\frac{3 v_{h}}{4}} 2 b \cdot e^{2} \cdot \frac{v_{h}\left(v_{h}-b\right)}{\left(2 b-v_{h}\right)^{3}} \cdot e^{-\frac{v_{h}}{2 b-v_{h}}} d b
$$

Substituting $t=v_{h} /\left(2 b-v_{h}\right)$, this becomes

$$
\frac{v_{h}^{2} e^{2}}{2} \int_{2}^{\infty}\left(1-\frac{1}{t}\right) e^{-t} d t=\frac{v_{h}}{4}\left(3-e^{2} \varphi(2)\right)
$$

where for any $z>0, \varphi(z)=\int_{z}^{\infty} \frac{e^{-t}}{t} d t$.

(ii) When $v_{h} / 2 \leq v_{l} \leq v_{h}$, the $L$ buyer wins the auction with probability $f\left(b_{l}\right)$ and the $H$ buyer wins when $b>b_{l}$. Therefore, the conditional expected selling price (given that $v_{h} / 2 \leq v_{h} \leq v_{h}$ ) is:

$$
\frac{2}{v_{h}} \int_{\frac{v_{h}}{2}}^{v_{h}}\left(b_{l} F\left(b_{l}\right)+\int_{b_{l}}^{\frac{3 v_{h}}{4}} b f(b) d b\right) d v_{l}
$$

The first integral is:

$$
\frac{2}{v_{h}} \int_{\frac{v_{h}}{2}}^{v_{h}} b_{l} F\left(b_{l}\right) d v_{l}=2 \int_{\frac{v_{h}}{2}}^{v_{h}}\left[\left(1-\frac{v_{h}}{4 v_{l}}\right) \cdot \frac{e^{2}}{2} \cdot \frac{2 v_{l}}{2 v_{l}-v_{h}} \cdot e^{-\frac{2 v_{l}}{2 v_{l}-v_{h}}}\right] d v_{l}
$$


By substituting $t=v_{h} /\left(2 v_{l}-v_{h}\right)$ and integrating by parts this becomes:

$$
\frac{e \cdot v_{h}}{4} \int_{1}^{\infty}\left(\frac{2}{t^{2}}+\frac{1}{t}\right) e^{-t} d t=\frac{v_{h}}{4}(2-e \varphi(1))
$$

The second integral, which requires a more lengthy and careful treatment is computed in the same manner:

$$
\frac{2}{v_{h}} \int_{\frac{v_{h}}{2}}^{v_{h}}\left(\int_{b_{l}}^{\frac{3 v_{h}}{4}} b f(b) d b\right) d v_{l}=\frac{v_{h}}{4}\left(1+e^{2} \varphi(2)\right)
$$

Combining both integrals yields the expected selling price (given $v_{l} \geq v_{h}$ ) is

$$
\frac{v_{h}}{4}\left(3-e \cdot \varphi(1)+e^{2} \cdot \varphi(2)\right)
$$

Since $P\left\{0 \leq v_{l} \leq v_{h} / 2\right\}=P\left\{v_{h} / 2 \leq v_{l} \leq v_{h}\right\}=1 / 2$, using (11) and (12), the expected selling price is:

$$
\frac{1}{4} \int_{0}^{1}\left(3-\frac{e}{2} \varphi(1)\right) v_{h}\left(2 v_{h}\right) d v_{h}=\frac{1}{2}-e \cdot \frac{\varphi(1)}{12} \approx 0.450304
$$

The welfare loss is equal to

$$
\int_{0}^{1} \frac{1}{v_{h}} \int_{\frac{v_{h}}{2}}^{v_{h}} F\left(b_{l}\right)\left(v_{h}-v_{l}\right) d v_{l}\left(2 v_{h}\right) d v_{h}=e \cdot \varphi(1) / 24
$$

We next calculate the low-value buyer's profits. The low-value buyer has expected profits equal to

$$
\int_{0}^{1} \frac{1}{v_{h}} \int_{\frac{v_{h}}{2}}^{v_{h}} F\left(b_{l}\right)\left(v_{l}-b_{l}\right) d v_{l}\left(2 v_{h}\right) d v_{h}=e \cdot \varphi(1) / 24
$$

Now we calculate the high-value buyer's profits. The total surplus in the environment is $2 / 3$. This should equal the sum of the seller's surplus, low-value buyer's surplus, the high-value buyer's surplus and the welfare loss.

From this we find that the high-value buyer's expected profits is simply 1/6. 